\title{
Trajectory Design Combining Invariant Manifolds with Discrete Mechanics and Optimal Control
}

\author{
Ashley Moore* \\ California Institute of Technology, Pasadena, California 91125 \\ Sina Ober-Blöbaum $\ddagger$ \\ University of Paderborn, 33098 Paderborn, Germany \\ and \\ Jerrold E. Marsden \\ California Institute of Technology, Pasadena, California 91125 \\ DOI: $\underline{10.2514 / 1.55426}$
}

\begin{abstract}
A mission design technique that combines invariant manifold techniques, discrete mechanics, and optimal control produces locally optimal low-energy trajectories. Previously, invariant manifolds of the planar circular restricted three-body problem have been used to design trajectories with relatively small midcourse change in velocity $\Delta V$. A different method of using invariant manifolds is explored to design trajectories directly in the four-body problem. Then, using the local optimal control method DMOC (Discrete Mechanics and Optimal Control), it is possible to reduce the midcourse $\Delta V$ to zero. The influence of different boundary conditions on the optimal trajectory is also demonstrated. These methods are tested on a trajectory that begins in Earth orbit and ends in ballistic capture at the moon. Impulsive DMOC trajectories require up to $19 \%$ less $\Delta V$ than trajectories using a Hohmann transfer. When applied to low-thrust trajectories, DMOC produces an improvement of up to $59 \%$ in the mass fraction and $22 \%$ in travel time when compared with results from shooting methods.
\end{abstract}

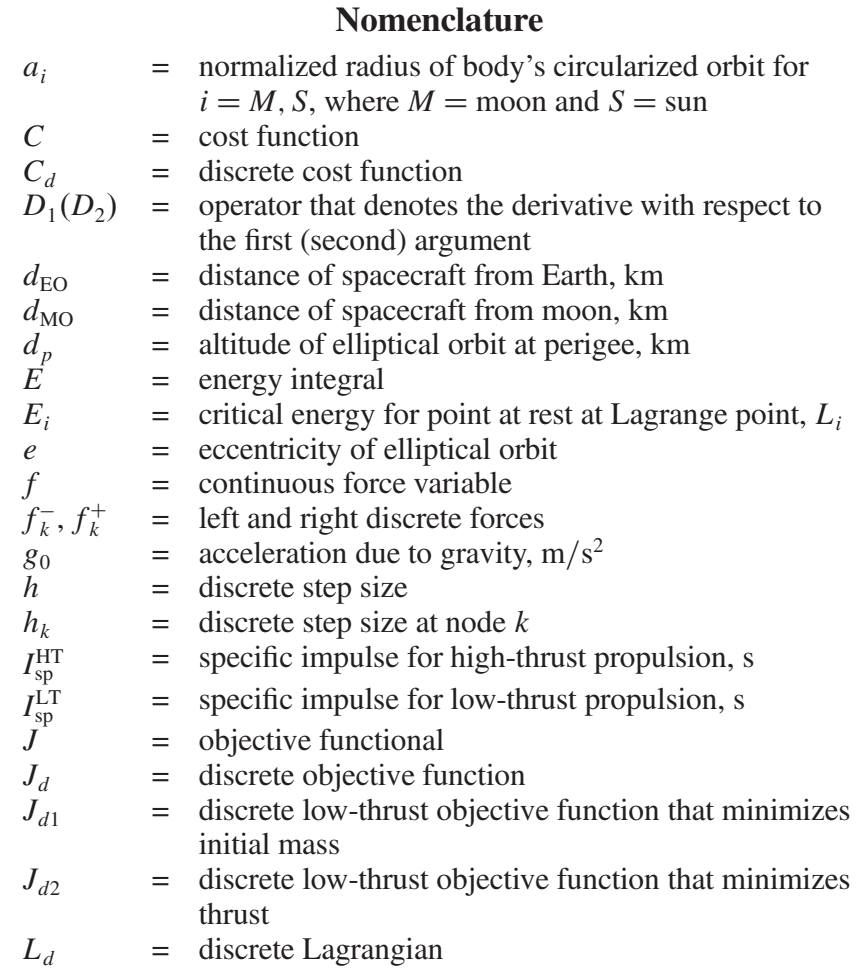

Received 9 July 2011; accepted for publication 13 February 2012. Copyright $\odot 2012$ by the American Institute of Aeronautics and Astronautics, Inc. All rights reserved. Copies of this paper may be made for personal or internal use, on condition that the copier pay the $\$ 10.00$ per-copy fee to the Copyright Clearance Center, Inc., 222 Rosewood Drive, Danvers, MA 01923; include the code 0731-5090/12 and \$10.00 in correspondence with the CCC.

*Ph.D. Graduate, Control and Dynamical Systems, MC 107-81.

$\dagger$ Junior Professor, Computational Dynamics and Optimal Control; sinaob@math.upb.de.

Carl F. Braun Professor of Engineering and CDS, Control and Dynamical Systems, MC 107-81; deceased.
$L_{i} \quad=\quad$ Lagrange point for $i=1, \ldots, 5$, equilibrium points of the planar circular restricted three-body problem

$\mathcal{M} \quad=$ energy surface

$M_{i} \quad=$ mass of body, $i=E, S, M$, where $E=$ Earth, $S=$ sun, and $M=$ moon, $\mathrm{kg}$

$m \quad=$ propellant mass, $\mathrm{kg}$

$m_{i} \quad=$ normalized mass of body $i, i=S, E, M$

$m_{p} \quad=$ mass consumption, $\mathrm{kg}$

$P \quad=$ spacecraft

$\mathcal{Q} \quad=$ configuration space

$q=$ configuration variable

$q_{k} \quad=$ discrete configuration variable

$r_{a}, r_{p}=$ radius of elliptical orbit at apoapsis and perigee, $\mathrm{km}$

$r_{M_{H}}, a_{H}=$ perigee radius, semimajor axis of Hohmann transfer ellipse, $\mathrm{km}$

$T=$ low-thrust magnitude, $\mathrm{N}$

$T_{k}=$ discrete thrust magnitude applied at node $k, \mathrm{~N}$

$T_{\max }=$ maximum allowable thrust, $\mathrm{N}$

$T Q=$ state space

$T_{t} \quad=$ normalized time denoting transition from low thrust to no thrust

$T_{x, k} \quad=$ discrete thrust at node $k$ in $x$ direction, $\mathrm{N}$

$T_{y, k} \quad=$ discrete thrust at node $k$ in $y$ direction, $\mathrm{N}$

$t_{k}=$ discrete time variable

$u=$ continuous control parameter

$u_{k} \quad=$ discrete control variable

$u_{x} \quad=$ control force in $x$ direction

$u_{y} \quad=$ control force in $y$ direction

$v_{r} \quad=$ normalized radial velocity

$v_{x, k}=$ normalized discrete velocity at node $k$ in $x$ direction

$v_{y, k}=$ normalized discrete velocity at node $k$ in $y$ direction

$x_{i}, y_{i}=x, y$ position of body $i$ for $i=E, S, M$

$\alpha_{V} \quad=$ scales nondimensional velocity to meters per second

$\Delta V=$ magnitude of change in velocity, $\mathrm{m} / \mathrm{s}$ or $\mathrm{km} / \mathrm{s}$

$\Delta V_{C}=\Delta V$ necessary to enter elliptical capture orbit at moon, $\mathrm{km} / \mathrm{s}$

$\Delta V_{H} \quad=\Delta V$ necessary to leave Earth orbit using a Hohmann transfer, $\mathrm{km} / \mathrm{s}$ 


$\begin{array}{lll}\Delta V_{i} & = & \Delta V \text { for circular orbit insertion at body } i, i=E, M, \\ & \mathrm{~m} / \mathrm{s} \text { or km/s } \\ \Delta V_{\text {traj }}= & \Delta V \text { applied throughout trajectory, } \mathrm{m} / \mathrm{s} \\ \Delta t & = & \text { discrete time grid } \\ \Delta \tilde{t} & = & \text { refined discrete time grid } \\ \delta & = & \text { variational operator } \\ \delta q_{k} & = & \text { discrete configuration variation } \\ \theta_{i} & = & \text { angle of body } i \text { with respect to } x \text { axis of coordinate } \\ & = & \text { system for } i=M, S, \text { rad } \\ \mu & = & \text { mass parameter of planar circular restricted } \\ & = & \text { discrete thrust optimization variable, } \mathrm{N} \\ \tau_{k} & = & \text { angle of semimajor axis of elliptical orbit at the } \\ \Phi & = & \text { moon with respect to the Earth-moon } x \text { axis, deg } \\ & & \text { sun-Earth frame, deg frame with respect to } \\ \phi & = & \text { effective potential in rotating frame } \\ \Omega & = & \text { normalized rotation rate of body } i \text { for } i=M, S \\ \omega_{i} & \end{array}$

\section{Introduction}

M ANY techniques focus on the design of spacecraft trajectories. For example, invariant manifolds of the planar circular restricted three-body problem (PCR3BP) can be used to find energy efficient trajectories that follow the natural dynamics of the solar system from one region of space to another. The three-body problem is well understood and allows for the design of complicated trajectories not possible using patched conics. Because the $N$-body problem is notoriously difficult to solve, much work has focused on patching multiple three-body systems together, which typically include impulsive control at the intersection of the invariant manifolds of the two systems. This work aims to extend that idea, to design a trajectory using four-body dynamics, and to apply local optimal control throughout the trajectory. The combination of invariant manifold techniques in the PCR3BP and the optimal control algorithm DMOC (Discrete Mechanics and Optimal Control) facilitates the design of minimal $\Delta V$ trajectories in the planar fourbody problem.

Since the 1950s, countless missions have targeted the moon, sending spacecraft along trajectories for flybys, lunar observation orbits, and both manned and unmanned lunar landings. More recently, propulsion technology and design techniques, including the use of invariant manifolds, have facilitated the design of creative fuelefficient trajectories. For example, in an effort to salvage Japan's Hiten mission, Belbruno and Miller [1,2] designed an unusual and fuel-efficient trajectory using invariant manifolds that resulted in ballistic capture at the moon. In addition, ESA's SMART-1 [3], was launched in 2003 to demonstrate the potential use of ion propulsion for future interplanetary and deep-space missions. The sustained thrust provided by the ion thruster allowed the spacecraft to spiral out from an elliptical orbit around the Earth to the moon and then spiral in for lunar capture.

This work presents a method for the design of locally optimal trajectories from the Earth to the moon in the planar four-body problem. First, initial guess trajectories are created using two different approaches based on invariant manifolds of the PCR3BP. The first method replicates a trajectory similar to the Shoot the Moon trajectory presented by Koon et al. [4]. Their paper describes the design of a trajectory that begins in low Earth orbit, travels along the invariant manifolds of the sun-Earth (SE) and Earth-moon (EM) PCR3BPs, and ends in ballistic capture at the moon, using a total $\Delta V$ of approximately $3245 \mathrm{~m} / \mathrm{s}$ (3211 m/s to leave Earth orbit and $34 \mathrm{~m} / \mathrm{s}$ applied midcourse). Mingotti et al. [5] describe a low-thrust version of this trajectory. The second method explores a different way of exploiting the manifold structure directly in the planar fourbody problem. Both trajectories are used as initial guesses for DMOC, which searches for a locally optimal trajectory in the fourbody system, applying control throughout the trajectory to decrease the total $\Delta V$.

\section{A. Invariant Manifolds}

Invariant manifolds are tubelike structures along which a spacecraft may travel, expending no energy. The manifolds can lead, for example, to periodic orbits around the Lagrange points of the PCR3BP. Conley [6] and McGehee [7] were the first to study the orbit structures around the $L_{1}$ and $L_{2}$ Lagrange points, classifying the trajectories as asymptotic orbits that asymptotically approach the periodic orbit, transit orbits that cross the equilibrium region around the Lagrange point from one region to another, or nontransit orbits. They also considered how to use such orbits to travel from the Earth to the moon. The transport made possible by invariant manifolds has been exploited for several different trajectories. Marsden and Ross [8] and Koon et al. [9] offer a great overview of the subject. More specifically, transfer from the Earth to periodic orbits around $L_{1}$ and $L_{2}$ is described by Barden et al. [10] and Gómez et al. [11]. The formal existence of heteroclinic connections between periodic orbits of the same energy is investigated by Koon et al. [12].

Invariant manifolds are particularly useful for the design of trajectories from the Earth to the moon, in the Jovian moon system, and in the Saturian moon system. The design of a multimoon orbiter in the Jupiter system is studied in Gómez et al. [13]. The combination of resonance and gravity assists is used for trajectories to Titan in [14,15] and in the Jupiter system by Ross and Scheeres [16]. Resonance and heteroclinic connections are shown to explain the motion of some comets, particularly those around Jupiter, presented by Koon et al. [17].

In addition to the Hiten mission described earlier, other missions have taken advantage of invariant manifolds. For example, the Genesis Discovery Mission successfully flew on a trajectory following the invariant manifolds of the sun-Earth system along a heteroclinic connection between $L_{1}$ and $L_{2}$, as presented by Wilson et al. [18] and later described in Koon et al. [19]. Central to the research presented here is the work of Koon et al. $[4,20]$ on the Shoot the Moon problem, a low-energy transfer from the Earth to the moon facilitated by the patched three-body problem of the sun-Earth and Earth-moon systems. This problem was also studied by Howell and Kakoi [21] and Mingotti and Topputo [22]. Lo [23] proposes how invariant manifolds and their connections that make up the Interplanetary Superhighway may be used for future NASA missions as part of the Origins Program.

Invariant manifold techniques typically lead to trajectories that require some kind of control, whether it be impulsive or low thrust. As mentioned earlier, Mingotti et al. [5] explore the use of low thrust combined with invariant manifolds to reach an elliptical orbit at the moon. Lo et al. [24] further examines the role of invariant manifolds for low-thrust trajectory design. Davis et al. [25] proposes an optimization technique for connecting periodic orbits about $L_{1}$ and $L_{2}$ of different energy. Marchand et al. [26] explores the use of optimal control for spacecraft formation keeping in orbits near $L_{1}$ and $L_{2}$.

An extension of invariant manifold techniques to account for a continuously applied control force is presented in Dellnitz et al. [27] and employed to design a trajectory from Earth to Venus and from Earth to $L_{2}$ in [28]. However, so far, techniques like this are only computationally reasonable for a constant one-dimensional control force. The research presented here employs a time-dependent control law influencing all degrees of freedom of the spacecraft at each time node that are optimal with respect to a certain goal. Therefore, the application of a local optimal control scheme is indispensable for the design of trajectories with more complex control laws. The thrustless trajectories designed using invariant manifold techniques serve as initial guesses for the optimization of the controlled model.

\section{B. Local Optimal Control}

Optimal control methods have been applied to many different space-related problems already. For example, in Junge et al. [29], DMOC is used to optimally raise a spacecraft in circular orbit to an orbit of greater radius and to reconfigure a group of hovercraft. Trajectory design in the four-body problem is a natural extension for DMOC. DMOC is a direct local optimal control method based on the 


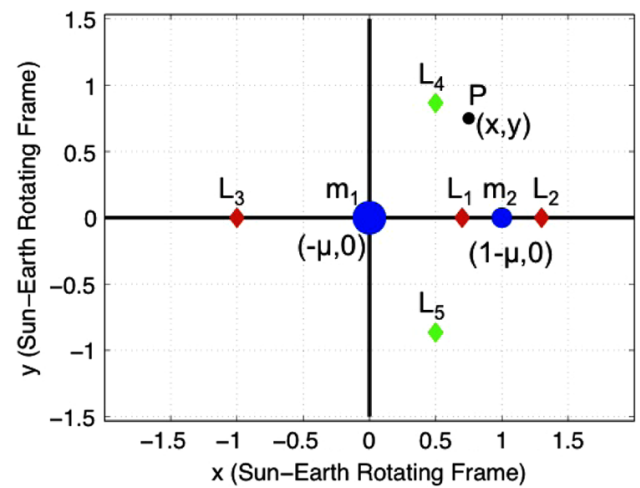

Fig. 1 Geometry of PCR3BP in sun-Earth rotating frame with two primary masses, $m_{1}$ and $m_{2}$, and Lagrange points $\left\{L_{i}\right\}_{i=1}^{5}$.

discretization of the variational structure of the mechanical system directly. The discretization of the Lagrange-d'Alembert principle [30] leads to structure-preserving time-stepping equations, which serve as equality constraints for the resulting finite dimensional nonlinear optimization problem [31]. This problem can be solved by standard nonlinear optimization techniques such as sequential quadratic programming (see, e.g., Gill et al. [32,33], Powell [34], and Han [35]).

This paper begins with background in Sec. II, describing the use of invariant manifolds of the three-body problem for trajectory design, the four-body model used for optimization, and DMOC. Next, the two different methods for designing an initial guess trajectory are presented in Sec. III before providing details on the optimization process, including the constraints, objective function, and results in Sec. IV. In Sec. V, the paper proceeds with a description of the Shoot the $\overline{M o o n}$ problem in the low-thrust framework. All optimization results are compared with trajectories found in the literature in Sec. VI. Ideas for future work and a summary of the major findings conclude the paper.

\section{Background}

The fundamental theory necessary for this work is presented here, including invariant manifolds of the planar circular restricted threebody problem, a controlled four-body model, and DMOC.

\section{A. Invariant Manifolds of the Three-Body Problem}

Trajectory design using invariant manifolds begins with two coupled planar circular restricted three-body problems [20]. The geometry of the PCR3BP is shown in Fig. 1. For each PCR3BP, the motion of a body is described under the influence of two main bodies, either the sun and the Earth in the sun-Earth system, or the Earth and the moon for the Earth-moon system. Each system is described in a rotating coordinate frame, and mass is normalized with the mass parameter

$$
\mu=\frac{M_{2}}{M_{1}+M_{2}}
$$

where $M_{1}>M_{2}$. For example, in the sun-Earth three-body system, $M_{1}$ denotes the mass of the sun in kilograms, and $M_{2}$ denotes the mass of the Earth in kilograms. The normalized mass of the larger body is denoted by $m_{1}=1-\mu$, and the normalized mass of the smaller body is $m_{2}=\mu$. The two primary bodies rotate in circular, planar orbits about their common center of mass at the origin. The third body, the spacecraft, is assumed to have infinitesimal mass. The primary bodies, $m_{1}$ and $m_{2}$, are positioned at $(-\mu, 0)$ and $(1-\mu, 0)$, respectively. The equations of motion for the PCR3BP are

$$
\begin{aligned}
& \ddot{x}-2 \dot{y}=\frac{\partial \Omega}{\partial x} \\
& \ddot{y}+2 \dot{x}=\frac{\partial \Omega}{\partial y}
\end{aligned}
$$

where

$$
\Omega=\frac{x^{2}+y^{2}}{2}+\frac{1-\mu}{\sqrt{(x+\mu)^{2}+y^{2}}}+\frac{\mu}{\sqrt{(x-1+\mu)^{2}+y^{2}}}
$$

The system, Eqs. (2-4), has five equilibrium points $L_{1}, \ldots, L_{5}$ (see Fig. 1); the unstable $\bar{L}_{2}$ point is of interest for this work.

Stable and unstable manifolds emanate from the periodic orbit of the $L_{2}$ Lagrange point, shown in Fig. 2. A method for computing invariant manifolds is described in Barden et al. [10] and Ross [36]. These manifold tubes control transport into and out of the region around $m_{2}$ [20]. The unstable manifold of the sun-Earth system leads away from the periodic orbit around $L_{2}$, whereas the stable manifold leads toward the periodic orbit as time flows forward.

The equations of motion for the PCR3BP are Hamiltonian and time independent, so there exists the following energy integral

$$
E=\frac{1}{2}\left(\dot{x}^{2}+\dot{y}^{2}\right)-\Omega(x, y)
$$

The phase space of the PCR3BP may be divided into regions of possible and forbidden motion based on this energy [36]. There are five possible cases, with the first four cases shown in Fig. 3 . Each plot shows the Hill's region, a projection of the energy surface $\mathcal{M}(\mu, e)=\{(x, y, \dot{x}, \dot{y}) \mid E(x, y, \dot{x}, \dot{y})=e\}$ onto configuration space, for a particular energy level. The cases are distinguished by the critical energy $\left\{E_{i}\right\}_{i=1}^{5}$, which represents the energy of a particle at rest at the Lagrange point $\left\{L_{i}\right\}_{i=1}^{5}$. For example, if the energy of the

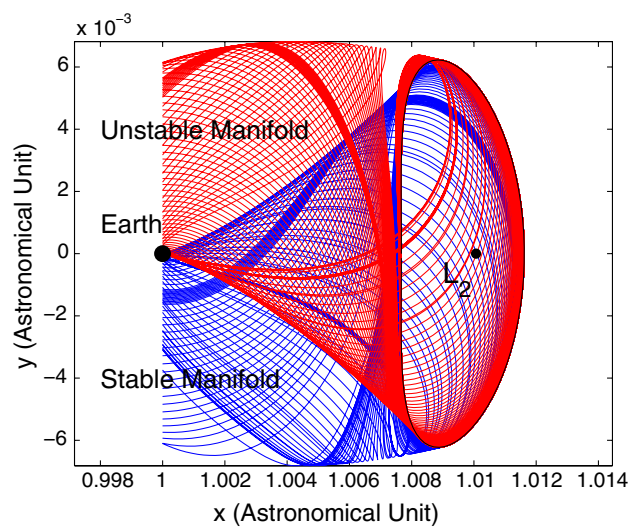

a)

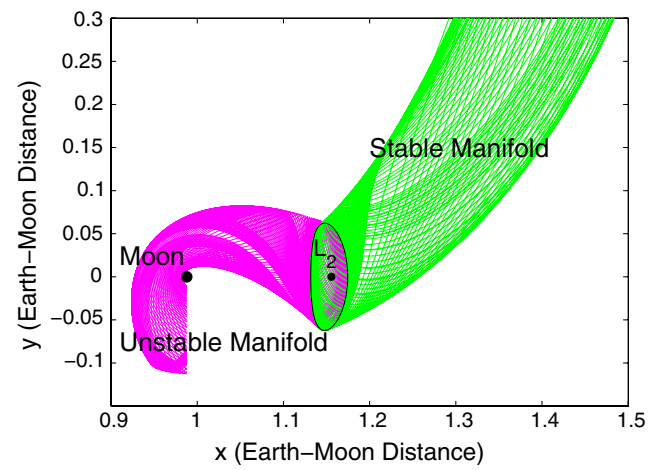

b)

Fig. 2 Manifolds emanate from the periodic orbit about $L_{2}$. a) Stable and unstable manifolds of the sun-Earth $L_{2}$ Lagrange point. b) Stable and unstable manifolds of Earth-moon $L_{2}$ Lagrange point. 

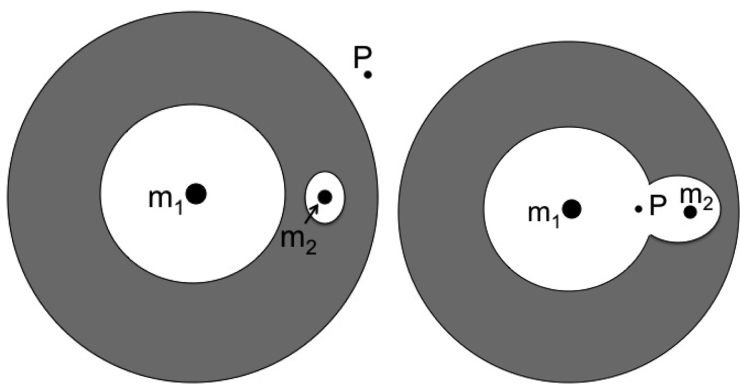

a) $E<E_{1}$

b) $E_{1}<E<E_{2}$
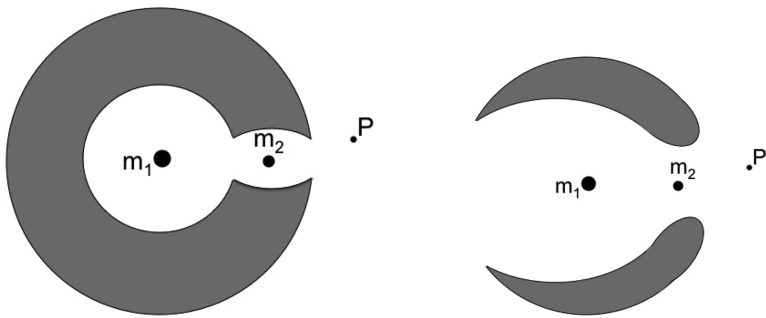

c) $E_{2}<E<E_{3}$

d) $E_{3}<E<E_{4}=E_{5}$

Fig. 3 Regions of possible motion: a) $P$ cannot move between $m_{1}$ and $m_{2}$, b) $P$ can move between $m_{1}$ and $m_{2}$ via $L_{1}$, c) $P$ may move from $m_{1}$ to $m_{2}$ to exterior region via $L_{1}$ and $L_{2}$, and d) $P$ may also travel past $m_{1}$ to exterior region via $L_{3}$. Case $5 E>E_{5}$ is not shown: $P$ may move freely in $x-y$ plane.

spacecraft is greater than $E_{2}$ but less than $E_{3}$, it is energetically possible for the spacecraft to move through the manifold tubes from the region surrounding $m_{2}$ to an exterior region and vice versa, as shown in Fig. 3c. Furthermore, this energy is important for transfer between manifolds of different PCR3BPs.

\section{B. Controlled Four-Body Model}

Optimization is used to refine the initial guess trajectory in a controlled four-body model, minimizing the control effort that translates to a minimized $\Delta V$. The four-body model used here is modeled in the sun-Earth rotating frame, similar to the PCR3BP, with the moon as a perturbation. The control force is denoted by $u=\left(u_{x}, u_{y}\right)$ where $u_{x}$ is the control force in the $x$ direction and $u_{y}$ is the control force in the $y$ direction.

Using the coordinates of the PCR3BP, the $x$ coordinates of the trajectory vary between $(0.995,1.01)$, whereas the $y$ coordinates vary between $(-0.006,0.006)$. The difference in scale between these numbers can cause problems for the optimization; the convergence improves when both $x$ and $y$ variables hover around the origin [37]. Therefore, a simple change of coordinates shifts the Earth so that it is centered at the origin. In general, the shifted problem converges faster and with smaller constraint residuals than the original problem.

This controlled four-body model describes the dynamics of the sun, Earth, moon, and spacecraft such that the moon rotates around the Earth's center in planar circular motion. Then, the Earth and sun rotate in planar circular motion about their center of mass. As before, the mass of the spacecraft is negligible. Figure 4 shows the geometry of this four-body model. The controlled equations of motion for this model in sun-Earth rotating coordinates are [36]

$$
\begin{aligned}
& \ddot{x}-2 \dot{y}=\frac{\partial \Omega}{\partial x}+u_{x} \\
& \ddot{y}+2 \dot{x}=\frac{\partial \Omega}{\partial y}+u_{y}
\end{aligned}
$$

where

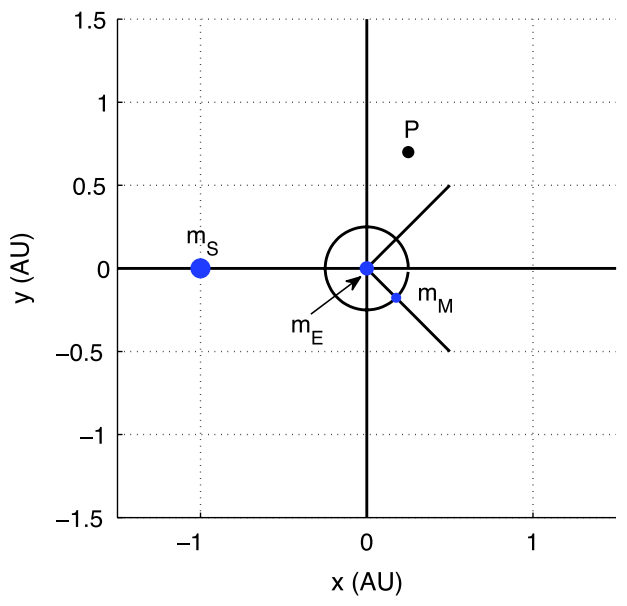

Fig. 4 Controlled four-body model: geometry in the sun-Earth rotating frame with three primary masses $m_{S}, m_{E}$, and $m_{M}$ and spacecraft $P$. The moon rotates relative to the sun-Earth rotating frame, which is stationary.

$$
\begin{aligned}
\Omega & =\frac{x^{2}+y^{2}}{2}+\frac{m_{S}}{\sqrt{(x+1)^{2}+y^{2}}}+\frac{m_{E}}{\sqrt{x^{2}+y^{2}}} \\
& +\frac{m_{M}}{\sqrt{\left(x-x_{M}\right)^{2}+\left(y-y_{M}\right)^{2}}}
\end{aligned}
$$

and $m_{S}, m_{E}$, and $m_{M}$ are the normalized mass of the sun, Earth, and moon, respectively, given by

$$
\begin{gathered}
m_{S}=1-\mu \\
m_{E}=\mu
\end{gathered}
$$

$$
m_{M}=\frac{M_{M}}{M_{M}+M_{E}+M_{S}}=3.734 \times 10^{-8}
$$

and

$$
\mu=\frac{M_{E}}{M_{E}+M_{S}}=3.036 \times 10^{-6}
$$

Note that $M_{i}, i=E, M, S$, denotes the body's mass in kilograms. Also, $x_{M}$ and $y_{M}$ represent the $x$ and $y$ positions of the moon as a function of time given by

$$
\begin{gathered}
\theta_{M}=\omega_{M} t+\theta_{M 0} \\
x_{M}=a_{M} \cos \theta_{M} \\
y_{M}=a_{M} \sin \theta_{M}
\end{gathered}
$$

where $t$ is time, $\theta_{M 0}$ is the initial angle of the moon with respect to the $x$ axis in the sun-Earth rotating frame, $a_{M}=2.573 \times 10^{-3}$ is the normalized radius of the moon's circular orbit, and $\omega_{M}=12.369$ is the normalized rotation rate of the moon. As noted by Ross [36], the motion of the three bodies under this model is not dynamically coherent; the motion does not satisfy Newton's laws. However, the model provides an approximation to the real behavior and is effective for this problem.

\section{Discrete Mechanics and Optimal Control}

To compute a trajectory with minimal fuel consumption, local optimal control techniques are used. DMOC $[29,31]$ is an optimal control scheme based on a direct discretization of the Lagranged'Alembert principle of the mechanical system. The discretization 
leads to the forced discrete Euler-Lagrange equations, which are used as optimization constraints for a given objective function. The resulting restricted optimization problem is solved with a sequential quadratic programming (SQP) solver.

Consider a mechanical system to be moved along a curve $q(t) \in Q$ during the time interval $t \in\left[0, T_{f}\right]$ from an initial state $\left(q^{0}, \dot{q}^{0}\right)$ to a final state $\left(q^{T_{f}}, \dot{q}^{T_{f}}\right)$ under the influence of a force $f(q(t), \dot{q}(t), u(t))$ where $u(t) \in U$ is a control parameter. The curves $q$ and $u$ are chosen to minimize a given objective functional

$$
J(q, \dot{q}, u)=\int_{0}^{T_{f}} C(q(t), \dot{q}(t), f(q(t), \dot{q}(t), u(t))) \mathrm{d} t
$$

subject to the condition that the system satisfies the Lagranged'Alembert principle, which states that

$$
\delta \int_{0}^{T_{f}} L(q(t), \dot{q}(t)) \mathrm{d} t+\int_{0}^{T_{f}} f(q(t), \dot{q}(t), u(t)) \cdot \delta q(t) \mathrm{d} t=0
$$

for all variations $\delta q$ with $\delta q(0)=\delta q\left(T_{f}\right)=0$, where $L: T Q \rightarrow \mathbb{R}$ is the Lagrangian consisting of the kinetic minus potential energy of the system. For a more thorough treatment of the Lagrange-d'Alembert principle, see, for example, Marsden and Ratiu [38].

The optimal control problem stated in Eqs. (16) and (17) is now transformed into a finite dimensional constrained optimization problem using a global discretization of the states and the controls. The state space $T Q$ is replaced by $Q \times Q$ and the discretization grid is defined by $\Delta t=\left\{t_{k}=k h \mid k=0, \ldots, N\right\}, N h=T_{f}$, where $N$ is a positive integer and $h$ is the step size. The path $q:\left[0, T_{f}\right] \rightarrow Q$ is replaced by a discrete path $q_{d}:\left\{t_{k}\right\}_{k=0}^{N} \rightarrow Q$, where $q_{k}=q_{d}(k h)$ is an approximation to $q(k h)[\underline{30,31]}$. Similarly, the control path $u:\left[0, T_{f}\right] \rightarrow U$ is replaced by a discrete one. To this end, a refined grid $\Delta \tilde{t}$ is generated via a set of control points $0 \leq c_{1}<\cdots<c_{s} \leq 1$ and $\Delta \tilde{t}=\left\{t_{k \ell}=t_{k}+c_{\ell} h \mid k=0, \ldots, N-1 ; \ell=1, \ldots, s\right\}$. With this notation, the discrete control path is defined to be $u_{d}: \Delta \tilde{t} \rightarrow U$. The intermediate control samples $u_{k}$ on $\left[t_{k}, t_{k+1}\right]$ are defined as $u_{k}=\left(u_{k 1}, \ldots, u_{k s}\right) \in U^{s}$ to be the values of the control parameters guiding the system from $q_{k}=q_{d}\left(t_{k}\right)$ to $q_{k+1}=q_{d}\left(t_{k+1}\right)$, where $u_{k l}=u_{d}\left(t_{k l}\right)$ for $l \in\{1, \ldots, s\}$.

The discrete Lagrange-d'Alembert principle emerges using an approximation of the action integral in Eq. (17) by a discrete Lagrangian $L_{d}: Q \times Q \rightarrow \mathbb{R}$

$$
L_{d}\left(q_{k}, q_{k+1}\right) \approx \int_{k h}^{(k+1) h} L(q(t), \dot{q}(t)) \mathrm{d} t
$$

and discrete forces

$$
f_{k}^{-} \cdot \delta q_{k}+f_{k}^{+} \cdot \delta q_{k+1} \approx \int_{k h}^{(k+1) h} f(q(t), \dot{q}(t), u(t)) \cdot \delta q(t) \mathrm{d} t
$$

where the left and right discrete forces $f_{k}^{ \pm}$now depend on $\left(q_{k}, q_{k+1}, u_{k}\right)$. In the discrete framework, it is necessary to consider discrete paths $\left\{q_{k}\right\}_{k=0}^{N}$ such that, for all variations $\left\{\delta q_{k}\right\}_{k=0}^{N}$ with $\delta q_{0}=\delta q_{N}=0$, it is true that

$$
\delta \sum_{k=0}^{N-1} L_{d}\left(q_{k}, q_{k+1}\right)+\sum_{k=0}^{N-1}\left(f_{k}^{-} \cdot \delta q_{k}+f_{k}^{+} \cdot \delta q_{k+1}\right)=0
$$

In the same manner, an approximation of the objective functional, Eq. (16), generates the discrete objective function $J_{d}$ and discrete cost function $C_{d}$.

The goal of the discrete constrained optimization problem is to minimize the discrete objective function

$$
J_{d}\left(q_{d}, u_{d}\right)=\sum_{k=0}^{N-1} C_{d}\left(q_{k}, q_{k+1}, u_{k}\right)
$$

subject to the constraints

$$
\begin{gathered}
q_{0}=q^{0} \\
q_{N}=q^{T} \\
D_{2} L\left(q^{0}, \dot{q}^{0}\right)+D_{1} L_{d}\left(q_{0}, q_{1}\right)+f_{0}^{-}=0 \\
D_{2} L_{d}\left(q_{k-1}, q_{k}\right)+D_{1} L_{d}\left(q_{k}, q_{k+1}\right)+f_{k-1}^{+}+f_{k}^{-}=0 \\
-D_{2} L\left(q^{T}, \dot{q}^{T}\right)+D_{2} L_{d}\left(q_{N-1}, q_{N}\right)+f_{N-1}^{+}=0
\end{gathered}
$$

with $k=1, \ldots, N-1$. Note that $D_{1}\left(D_{2}\right)$ denotes the derivative with respect to the first (second) argument. The first two constraints require that the initial and final discrete positions match the continuous positions. The third and final constraints are the discrete momentum boundary conditions, and the fourth condition is the forced discrete Euler-Lagrange equation resulting from Eq. (20). The order of approximation of the discrete Lagrangian and the discrete forces given in Eqs. (18) and (19), respectively, determines the order of convergence of the optimal control scheme. In general, polynomial approximations to the trajectories and numerical quadrature are used to approximate the integrals. Then, the order of the discrete Lagrangian and the discrete forces is given by the order of the quadrature rule in use.

Balancing accuracy and efficiency, the discrete cost function $C_{d}$, the discrete Lagrangian $L_{d}$, and the discrete forces are approximated with the midpoint rule, and constant control parameters are assumed on each time interval with $l=1$ and $c_{1}=\frac{1}{2}$ :

$$
\begin{gathered}
C_{d}\left(q_{k}, q_{k+1}, u_{k}\right)=h C\left(\frac{q_{k+1}+q_{k}}{2}, \frac{q_{k+1}-q_{k}}{h}, u_{k}\right) \\
L_{d}\left(q_{k}, q_{k+1}\right)=h L\left(\frac{q_{k+1}+q_{k}}{2}, \frac{q_{k+1}-q_{k}}{h}\right) \\
f_{k}^{-}=f_{k}^{+}=\frac{h}{2} f\left(\frac{q_{k+1}+q_{k}}{2}, \frac{q_{k+1}-q_{k}}{h}, u_{k}\right)
\end{gathered}
$$

Equations (21-26) describe a nonlinear optimization problem with equality constraints, which can be solved by standard optimization methods like SQP. Optionally, inequality constraints on states and controls can be included. In contrast to other direct optimal control methods, DMOC is based on the discretization of the variational principle, Eq. (17), rather than a discretization of the ordinary differential equations. In Ober-Blöbaum et al. [39], a detailed analysis of DMOC resulting from this discrete variational approach is given. The optimization scheme is symplectic-momentum consistent, that is, the symplectic structure and the momentum maps corresponding to symmetry groups are consistent with the control forces for the discrete solution, independent of the step size $h$. Thus, the use of DMOC leads to a reasonable approximation to the continuous solution also for large step sizes (i.e., a small number of discretization points). However, other optimal control methods like collocation methods or shooting methods could be used as well (for an overview of these methods, see, e.g., $[\underline{40}, \underline{41}])$.

\section{Trajectory Design Using Invariant Manifolds}

This section focuses on two different methods that use invariant manifolds for trajectory design. Method 1, originally described with different results and cost function in Moore et al. [42], uses the unstable sun-Earth manifold and the stable Earth-moon manifold and begins with a trajectory designed in the three-body problem before modification in the four-body problem. Method 2 focuses on the stable sun-Earth manifold and the unstable Earth-moon manifold and directly generates a trajectory in the four-body problem. 


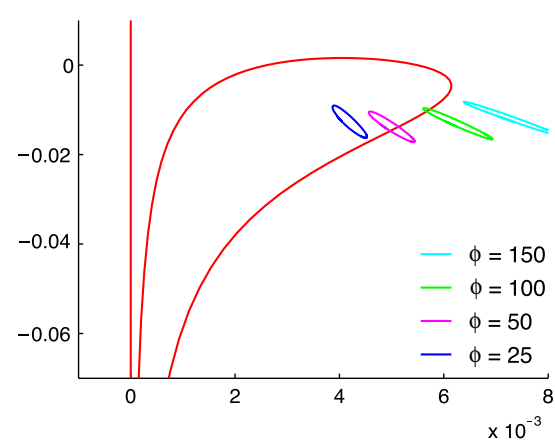

a) Intersection with phasing

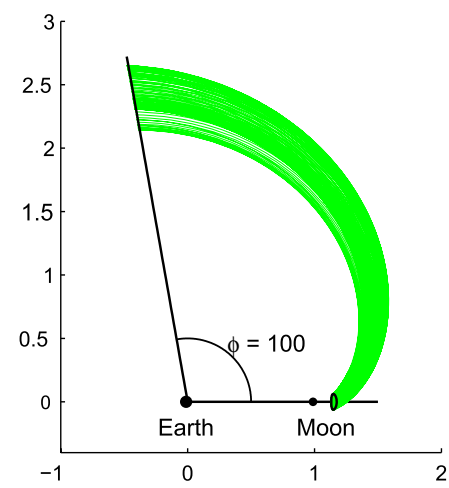

b) Earth-Moon stable manifold, $\phi=100$

Fig. 5 The phase $\phi$ of the Earth-moon line with respect to the sun-Earth line is varied until a suitable intersection of the sun-Earth unstable manifold (computed using the dynamics of the sun-Earth PCR3BP) and Earth-moon stable manifold (computed using the dynamics of the Earth-moon PCR3BP) is found.

\section{A. Method 1}

To achieve transfer between the Earth and moon using invariant manifolds, the first step is to locate an intersection of the unstable sun-Earth manifold with the stable Earth-moon manifold in which they do not overlap completely. A Poincaré section is used to find this intersection in the sun-Earth rotating frame. As shown in Fig. 5a, the phase $\phi$ of the Earth-moon frame with respect to the sun-Earth frame can be adjusted to identify such an intersection; $\phi=100 \mathrm{deg}$ is used here. Note that the manifolds do not intersect at all for $\phi=150 \mathrm{deg}$, whereas for $\phi=25 \mathrm{deg}$, at the intersection, the Earth-moon manifold lies entirely inside the sun-Earth manifold. Both situations are undesirable because they do not facilitate the desired transfer from the Earth to the moon. If the manifolds completely overlap, a trajectory originating in the intersection will flow through the sunEarth manifold, toward and then through the periodic orbit toward exterior space.

Using the Poincaré section, shown on the right-hand side of Fig. 6, a patch point is selected that falls within the stable manifold of the Earth-moon system and outside of, but very close to, the unstable manifold of the sun-Earth system. From the Poincare section, the patch point includes $x, y$, and $\dot{y}$. The $x$ velocity $\dot{x}$ is selected to ensure that the energy integral at the patch point equals that of the desired manifold. Forward integration of the conditions at the patch point $(x, y, \dot{x}, \dot{y})$ leads to a trajectory that flows along the stable Earthmoon manifold and ends in ballistic capture at the moon. Selection of the patch point just outside the sun-Earth invariant manifold guarantees that backward integration will generate a trajectory that flows along the invariant manifold and then bounces off the periodic orbit. To target the Earth, the initial conditions are modified slightly in $\dot{x}$ and $\dot{y}$. This modification is user dependent and requires much trial and error. First, very small changes are made to $\dot{x}$ and $\dot{y}$ to discern their effects. Gradually, the modifications are fine-tuned until a trajectory that travels within a desired distance of the Earth is found.

The sun-Earth and Earth-moon trajectories are patched together to form a trajectory that begins at the Earth and ends in ballistic capture at the moon. Ballistic capture here is defined to mean that the trajectory orbits the moon at least once within its sphere of influence. Note that, at the patch point, the energy is discontinuous; therefore, a $\Delta V$ is necessary to jump from the energy level of the sun-Earth manifold to the energy level of the Earth-moon manifold. For mathematical details about this process, see Koon et al. [20] and Ross [36]. The patched trajectory is shown in Fig. 7; it begins in a $135 \mathrm{~km}$ radius circular orbit about the Earth and ends in a 11,785 km circular orbit about the moon. An initial thrust of $3227.8 \mathrm{~m} / \mathrm{s}$ is required to escape Earth orbit along the trajectory, a midcourse $\Delta V$ of $60.6 \mathrm{~m} / \mathrm{s}$ is applied at the patch point, and a final $\Delta V$ of $197.8 \mathrm{~m} / \mathrm{s}$ is required to settle into a permanent circular orbit at the moon.

This trajectory is only valid for the patched three-body problem; therefore, it is necessary to modify it to fulfill the dynamics of the four-body problem. Beginning with the same initial conditions from the patch point, $\dot{x}$ and $\dot{y}$ are modified slightly and integrated using the four-body model. The modification is necessary due to the differences between the dynamics of the PCR3BP and the four-body problem. The patch point is modified differently for the sun-Earth section and the Earth-moon section because of the energy differences between the manifolds of the two systems. Thus, the initial conditions denoted by $\mathrm{IC}_{\mathrm{SE}}$ and $\mathrm{IC}_{\mathrm{EM}}$, respectively, can be expressed as

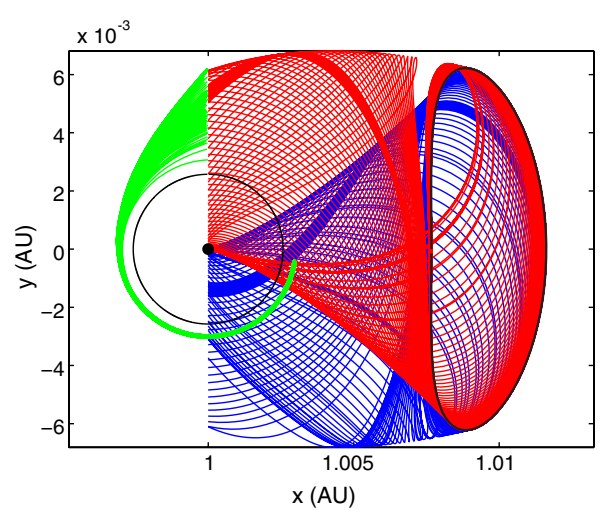

a) Manifolds

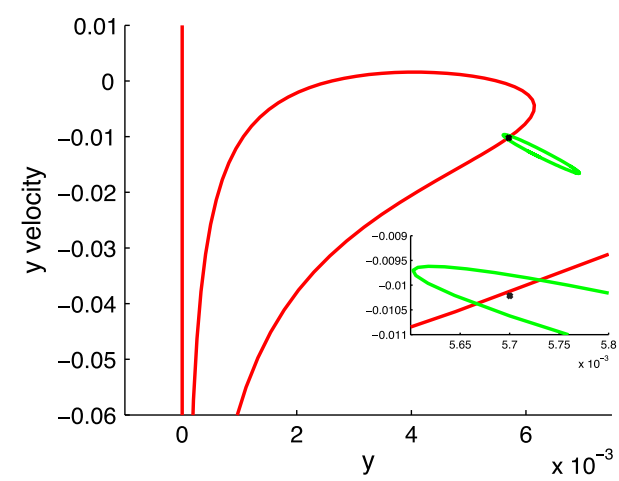

b) Poincaré Section

Fig. 6 a) Intersecting Earth-moon and sun-Earth manifolds in sun-Earth rotating coordinates. b) Poincaré section showing the intersection of the stable Earth-moon manifold with the unstable sun-Earth manifold. The patch point is chosen inside stable Earth-moon manifold and outside the unstable sun-Earth manifold. 


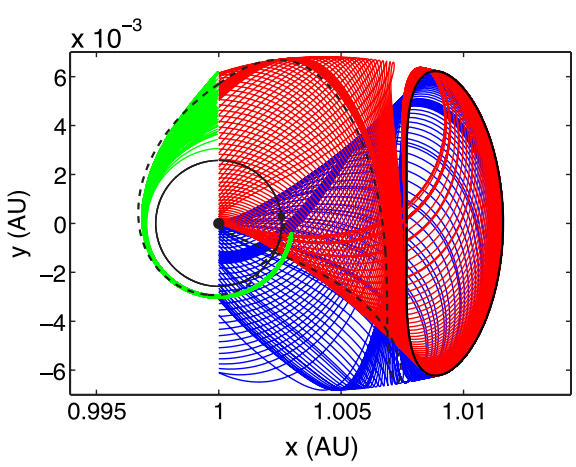

a) Trajectory in PCR3BP in SE Rotating Frame

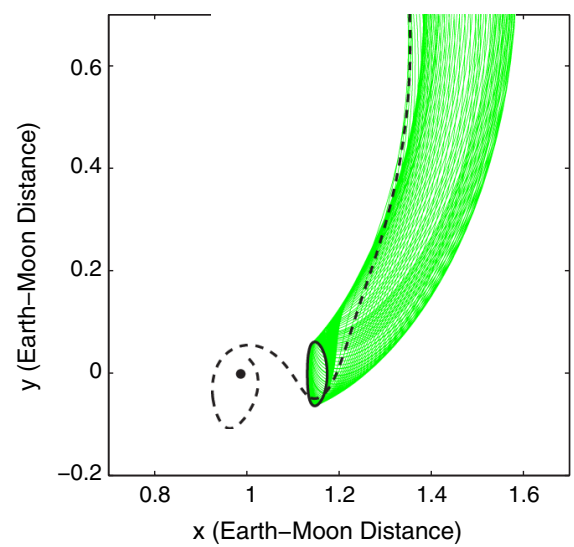

b) Capture at the Moon in EM Rotating Frame

Fig. 7 a) Trajectory in three-body problem (sun-Earth rotating coordinates) begins near the Earth, follows the sun-Earth unstable manifold toward the periodic orbit of $L_{2}$. It twists and then intersects the stable manifold of the Earth-moon system, following that manifold to the realm of the moon. b) Capture at the moon.

$$
\begin{gathered}
\mathrm{IC}_{\mathrm{SE}}=\left[\begin{array}{llll}
x & y & \dot{x}+\Delta \dot{x}_{\mathrm{SE}} & \dot{y}+\Delta \dot{y}_{\mathrm{SE}}
\end{array}\right] \\
\mathrm{IC}_{\mathrm{EM}}=\left[\begin{array}{llll}
x & y & \dot{x}+\Delta \dot{x}_{\mathrm{EM}} & \dot{y}+\Delta \dot{y}_{\mathrm{EM}}
\end{array}\right]
\end{gathered}
$$

$\mathrm{IC}_{\mathrm{SE}}$ is integrated backward to generate the sun-Earth portion of the trajectory, and $\mathrm{IC}_{\mathrm{EM}}$ is integrated forward to generate the Earthmoon portion of the trajectory. Note that the $\Delta$ values are adjusted manually until a good trajectory is found: a trajectory that begins and ends at a desired radius about the Earth and moon, respectively. Note that the initial and final momentum values may not be favorable. DMOC adjusts these momentum values according to the specified constraints and cost function during optimization. This trajectory serves as the initial guess for DMOC.

\section{B. Method 2}

To design a trajectory valid for the planar four-body problem, the process starts with the unstable Earth-moon manifold and stable sun-Earth manifold. A point $\mathrm{IC}_{M}$ is selected on the unstable Earthmoon manifold a desired distance from the moon. If this point is integrated backward in the Earth-moon PCR3BP, the trajectory would flow asymptotically to the periodic orbit. However, when integrated backward in the four-body problem (transformed to sunEarth rotating coordinates), the resulting trajectory Traj $_{M}$ flows from the moon toward, and then through, the Earth-moon $L_{2}$ periodic orbit. If the selected initial condition leads to a trajectory that bounces off the periodic orbit, modification of the velocity conditions should lead to the desired trajectory. For this example, such modification was not necessary. Next, another point $\mathrm{IC}_{E}$ is selected on the stable sun-Earth manifold some distance from the Earth. $\mathrm{IC}_{E}$ is integrated forward along the manifold using the sun-Earth four-body model, generating $\operatorname{Traj}_{E}$. In the sun-Earth PCR3BP, this trajectory would end on the periodic orbit; however, due to four-body perturbations, the trajectory bounces off the periodic orbit and flows back toward the moon's orbit. Figure 8a shows $\operatorname{Traj}_{E}$ in Earth-moon rotating coordinates, and Fig. $\underline{8 \mathrm{~b}}$ shows $\operatorname{Traj}_{E}$ in sun-Earth rotating coordinates.

Next, the intersection of the resulting trajectories in sun-Earth rotating coordinates is located; this is the patch point, shown in Fig. 8c. The conditions of $\mathrm{Traj}_{M}$ at the patch point are integrated forward in the four-body problem to create $\operatorname{Traj}_{M 2}$ (identical to $\mathrm{Traj}_{M}$, but it flows in the opposite direction, toward the moon instead of away from it). Note that, even though the trajectories intersect in $x-y$ space, they do not actually intersect in time or velocity. The discontinuity in velocity requires an impulsive $\Delta V$. The lack of intersection in time means that the position of the moon, given by Eqs. (13-15), is different for $\mathrm{Traj}_{M}$ and $\mathrm{Traj}_{E}$ and requires more consideration. Consequently, the position of the moon at the patch point for $\operatorname{Traj}_{M}$ is selected as the initial condition of the moon for both trajectories at the patch point. Because the moon is now in a different position than it was when $\operatorname{Traj}_{E}$ was devised, the velocity conditions at the patch point must be modified manually so that, when integrated backward, the resulting trajectory ends near the Earth, as desired. This trajectory is labeled $\operatorname{Traj}_{E 2}$. Figure $8 \mathrm{~d}$ shows the trajectory combining Traj $\operatorname{Tr}_{M 2}$ and $\operatorname{Traj}_{E 2}$ joined by an impulsive $\Delta V$ at the patch point. This trajectory serves as the initial guess for optimization. In Fig. 8, the manifolds are labeled such that $\mathrm{EM}_{U}\left(\mathrm{EM}_{S}\right)$ represents the Earth-moon unstable (stable) manifold, and $\mathrm{SE}_{U}\left(\mathrm{SE}_{S}\right)$ represents the sun-Earth unstable (stable) manifold.

Although the procedures for methods 1 and 2 appear complex and require a certain amount of user judgement, it is important to remember that the goal is to design a cohesive trajectory from the Earth to the moon. The invariant manifolds provide the main structure the trajectory follows, and modifications of the initial conditions simply fine-tune the trajectory, guiding it to a desired distance from the Earth or moon. As will be seen, if the trajectory begins near the Earth and ends near the moon, it is possible to further fine-tune the details during optimization, enforcing appropriate boundary conditions.

\section{Creation of Initial Guess}

When creating the initial guess using the two methods described in the preceding section, the nonlinearity of the dynamics poses a problem. Ideally, one step size would be used throughout the trajectory, but this leads to two possible and undesirable scenarios. First, if a medium step size is used, for example, $\mathcal{O}\left(10^{-2}\right)$, there are not enough nodes near the Earth and moon to accurately capture the dynamics, and general accuracy and numerical problems arise. On the other hand, if a sufficiently small step size is used, for example, $\mathcal{O}\left(10^{-5}\right)$, there are too many nodes leading to unreasonable computation time and memory problems. To solve this problem, the trajectory is broken into sections of different step size. For example, five sections are used for the initial guess found with method 1. Selecting the step size and number of nodes is an iterative, manual process that is repeated until a sufficiently accurate trajectory is produced. The variation in step size is accommodated in DMOC by supplying $h$ as a vector $h_{k}$, containing the step size at each node.

There are other approaches for designing the step size profile. For example, Betts and Huffman [43] describe an automated process for designing the time grid such that discretization errors in the solution are reduced. Moore et al. [44] compare Betts and Huffman's approach [43] with a similar method that aims to reduce discretization errors in the energy evolution and also examines the use of time adaptive variational integrators to generate an initial guess with continuous, variable step size profile.

The initial guess (IG) trajectories are plotted in Fig. 9. Two initial guess trajectories are created using each method: IG1 and IG2 are created using method 1, whereas IG3 and IG4 are created using 


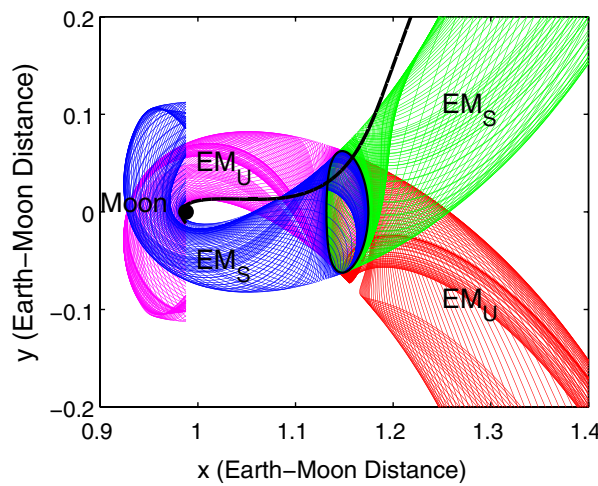

a) $\operatorname{Traj}_{M}$

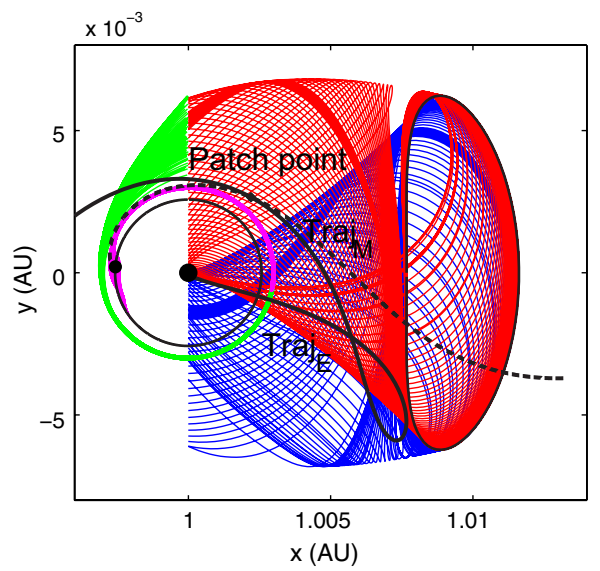

c) Intersection

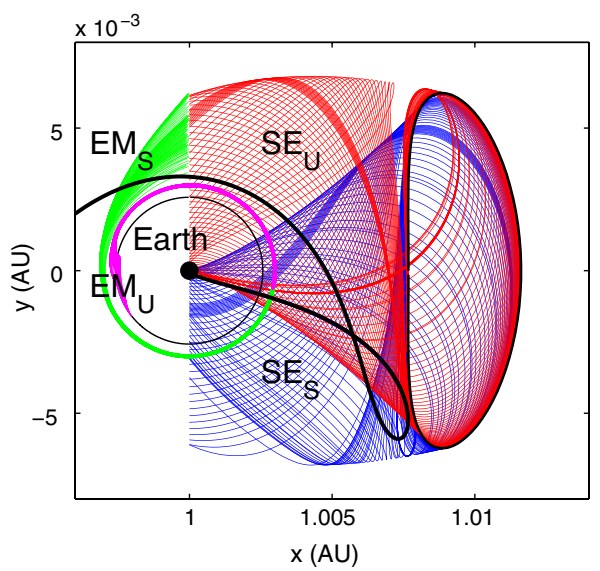

b) $\operatorname{Traj}_{E}$

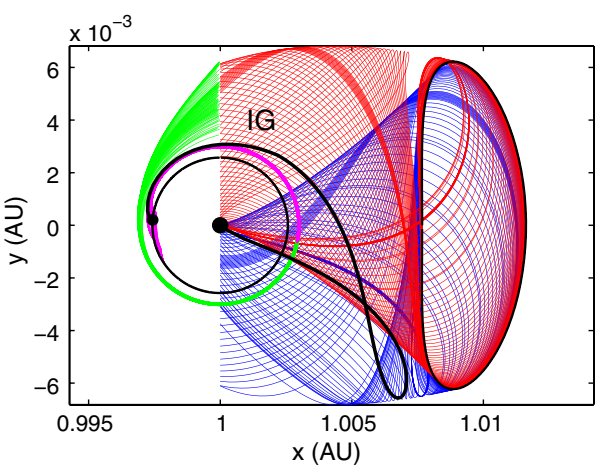

d) Combined Trajectory

Fig. 8 Process for method 2: a) Integrate point on Earth-moon unstable manifold backward in four-body problem. b) Integrate point on sun-Earth stable manifold forward in four-body problem. c) Locate intersection of the two trajectories; this is the new patch point. d) Integrate initial conditions at the patch point with consistent moon position to generate initial guess with impulsive $\Delta V$ at the patch point.

method 2. Note that IG3 and IG4 take roughly half the time to reach the moon compared with IG1 and IG2, and the final position of the moon is different for each trajectory. IG1 and IG2 are very similar except that IG1 ends at the first encounter with the moon. IG2 first loops around the moon (ballistic capture) and then continues until the spacecraft encounters the moon a second time. IG3 and IG4 differ most in the location of the patch point. In Fig. 9b, notice the strong kink in IG4; this is at the patch point. There is a larger discontinuity in velocity than at IG3's patch point.

Table 1 displays the trajectory details, including initial distance from the $\bar{E}$ arth, denoted $d_{\mathrm{EO}}$, final distance from the moon $d_{\mathrm{MO}}$, the total $\Delta V$, which is broken into $\Delta V_{E}$ (the $\Delta V$ necessary to leave circular Earth orbit), $\Delta V_{M}$ (the $\Delta V$ necessary to inject the spacecraft into a circular orbit at the moon), $\Delta V_{\text {traj }}$ (the $\Delta V$ applied throughout the trajectory, concentrated at the patch point for the initial guess), and the number of nodes. All four initial guesses begin within $500 \mathrm{~km}$ of the Earth, but they end at varying distances from the moon: $2614 \mathrm{~km}$ for IG1, $249 \mathrm{~km}$ for IG2, $685 \mathrm{~km}$ for IG3, and $267 \mathrm{~km}$ for IG4. Also, at 8683 and $9250 \mathrm{~m} / \mathrm{s}, \Delta V_{E}$ is much larger for IG3 and IG4. Additionally, the impulsive $\Delta V_{\text {traj }}$ at the patch point is 60,56 , 174, and $269 \mathrm{~m} / \mathrm{s}$ for IG1, IG2, IG3, and IG4, respectively. Note that IG3 and IG4 are quite poor as initial guess trajectories. In particular, $\Delta V_{E}$ is very large, and these trajectories will be viable only if optimization brings $\Delta V_{E}$ down to the more reasonable values of IG1 and IG2. These different initial guess trajectories will demonstrate the local nature of DMOC as well as the effect different constraints have on the optimal solution.

Note that the number of nodes used for each initial guess is different. As mentioned before, each trajectory is broken into sections of constant step size to accurately capture the dynamics while maintaining a reasonable number of nodes. Smaller step sizes are used near the Earth and moon, whereas larger step sizes are sufficient in regions far from the bodies. IG3 and IG4 use more nodes than IG1 and IG2, even though IG1 and IG2 are longer trajectories. The maximum step size used for IG3 and IG4 is 0.001, whereas the maximum step size used for IG1 and IG2 is 0.005 . The maximum step size is used in the middle portion of the trajectory, nearly in free space for IG1 and IG2, but closer to the Earth and the moon's orbit for IG3 and IG4. Therefore, the dynamics dictate a smaller maximum step size for IG3 and IG4, leading to more nodes.

Note that, even though the initial guesses have impulsive control applied at the patch point, when supplied to DMOC, the control force is assumed to be zero throughout the trajectory. This allows DMOC to find a solution with a smooth control profile. Because of the local nature of the SQP algorithm, if the impulsive force is included, DMOC returns a control profile with an impulse and much higher $\Delta V$.

\section{Optimization}

The optimization procedure begins with the formulation of cost function and constraints. Then, an SQP solver, SNOPT, performs the optimization. A number of optimization results are presented for the initial guesses described previously.

\section{A. Constraints and Objective Function}

The next step before the optimization is the formulation of constraints. The primary constraint enforces the system dynamics, requiring that the forced discrete Euler-Lagrange equations, Eq. (25), derived from the Lagrangian for the four-body model, are fulfilled. The Lagrangian is given by 


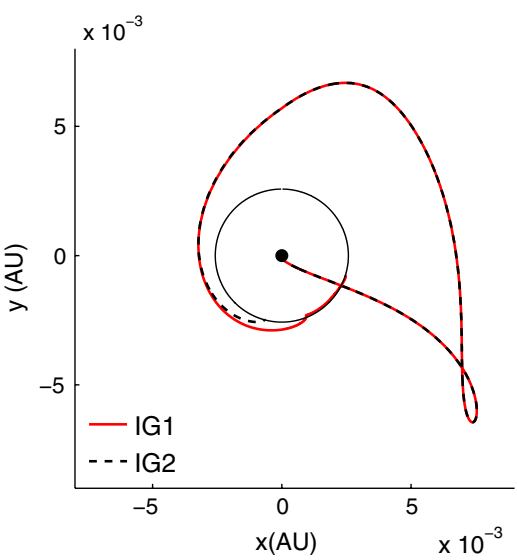

a) SM1 IG, SE rotating frame

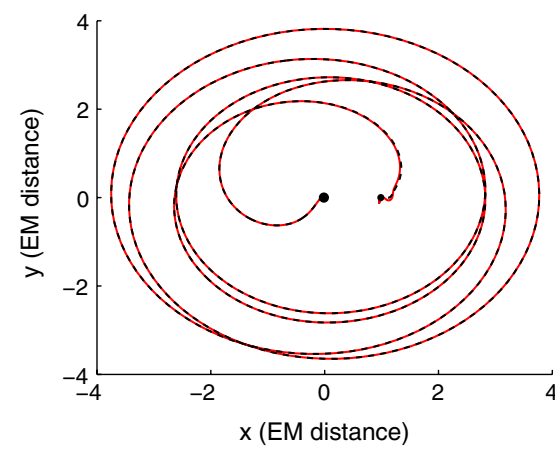

c) SM1 IG, EM rotating frame

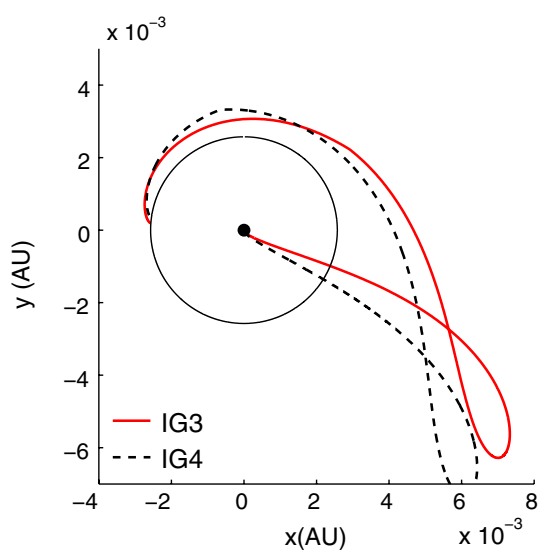

b) SM2 IG,SE rotating frame

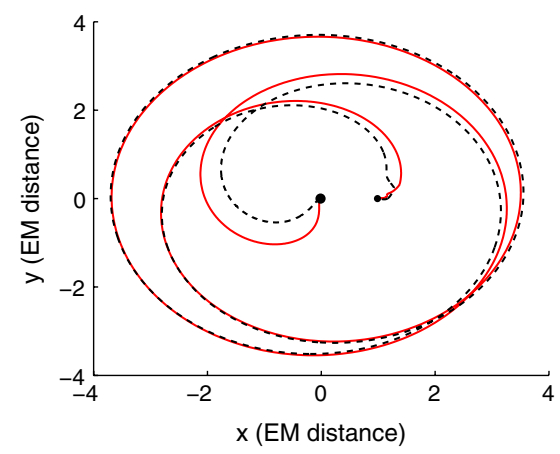

d) SM2 IG, EM rotating frame

Fig. 9 Initial guess trajectories: two initial guesses in sun-Earth rotating frame created using a) method 1 (IG1 and IG2) and b) method 2 (IG3 and IG4), Initial guesses in Earth-moon rotating frame for c) method 1 (IG1 and IG2) and d) method 2 (IG3 and IG4).

$$
\begin{aligned}
L= & \frac{1}{2}\left(\dot{x}^{2}+\dot{y}^{2}\right)+\frac{1}{2}\left(x^{2}+y^{2}\right)+x \dot{y}-y \dot{x}+\frac{m_{E}}{\sqrt{x^{2}+y^{2}}} \\
& +\frac{m_{S}}{\sqrt{(x+1)^{2}+y^{2}}}+\frac{m_{M}}{\sqrt{\left(x-x_{M}\right)^{2}+\left(y-y_{M}\right)^{2}}}
\end{aligned}
$$

The control force, $f(q, \dot{q}, u)=u$, consisting of control parameters $\left(u_{x}, u_{y}\right)$, represents the control force applied in the $x$ and $y$ direction, respectively, and is included in the forced discrete Euler-Lagrange equations.

In addition to the forced discrete Euler-Lagrange equations, a variety of boundary conditions are enforced, including the initial and final distance from the Earth and moon, respectively, the initial and final radial velocity, and a capture condition at the moon. For each optimization, the initial distance from the Earth is required to be $d_{\mathrm{EO}}=200 \mathrm{~km}$. The final distance from the moon is different for each optimization, either equaling that of the initial guess or being allowed to vary within some specified range. If the radial velocity is required to be zero, the velocity of the initial (final) node is tangential to the trajectory, which allows the spacecraft to move out of (into) a circular or elliptical orbit using less $\Delta V$. If the final distance from the moon is required to match that of the initial guess, ballistic capture happens

Table 1 Details of initial guess trajectories

\begin{tabular}{lrrrr}
\hline \hline & IG1 & IG2 & IG3 & IG4 \\
\hline Time of flight, days & 168 & 161 & 98 & 95 \\
Total $\Delta V, \mathrm{~m} / \mathrm{s}$ & 3966 & 3992 & 9951 & 10,193 \\
$\Delta V_{E}, \mathrm{~m} / \mathrm{s}$ & 3214 & 3214 & 8683 & 9250 \\
$\Delta V_{M}, \mathrm{~m} / \mathrm{s}$ & 692 & 722 & 1094 & 674 \\
$\Delta V_{\text {traj }}, \mathrm{m} / \mathrm{s}$ & 60 & 56 & 174 & 269 \\
$d_{\text {EO }}, \mathrm{km}$ & 195 & 195 & 217 & 378 \\
$\left.d_{\text {MO }}, \mathrm{km}\right)$ & 2614 & 249 & 685 & 267 \\
No. of nodes & 1810 & 1190 & 2271 & 3018 \\
\hline \hline
\end{tabular}

naturally. However, if the final altitude is allowed to vary, usually to decrease the final distance from that of the initial condition, ballistic capture may not be maintained by the optimization. As described by Belbruno and Miller [1], ballistic capture may be enforced with the condition

$$
E_{M}=\frac{1}{2}\left(\left(\dot{x}_{N}-\dot{x}_{M_{N}}\right)^{2}+\left(\dot{y}_{N}-\dot{y}_{M_{N}}\right)^{2}\right)-\frac{m_{M}}{r_{M_{N}}}<0
$$

where $\dot{x}_{N}$ and $\dot{y}_{N}$ are the $x$ - and $y$-velocity components of the spacecraft at the final node, and $\dot{x}_{M_{N}}$ and $\dot{y}_{M_{N}}$ are the velocity components of the moon at the final node. This equation gives the Keplerian energy with respect to the moon and states that the kinetic energy of the spacecraft relative to the moon at the final node must be less than the gravitational potential energy of the moon. Because of gravitational effects of the Earth and sun, this condition is not enough for permanent capture, but it does generate ballistic capture, as desired.

For each of the four initial guesses, two optimizations are performed with a slightly different set of constraints. Each optimal result is named according to the convention DMOC $i-j$, where $i=1, \ldots, 4$ is the initial guess number $(i=1,2$ correspond to method 1 and $i=3,4$ correspond to method 2), and $j=1,2$ is the optimization run number. For all the $j=1$ runs, the final distance from the moon is required to match that of the initial guess, except for DMOC 1-1 which is required to be $500 \mathrm{~km}$ instead of the $2615 \mathrm{~km}$ of the initial guess. Also, for these trials, the initial and final radial velocity must be zero. For all the $j=2$ runs, the final distance from the moon is allowed to vary within some range, only the initial radial velocity is zero, and the capture condition is enforced. The constraints for each run are outlined in Table 2.

The goal of the optimization is to minimize the control effort; correspondingly, the discrete objective function is 
Table 2 Constraints for each optimization run

\begin{tabular}{lcccc}
\hline \hline & $d_{\mathrm{EO}}, \mathrm{km}$ & $d_{\mathrm{MO}}, \mathrm{km}$ & $v_{r_{0}}=0$ & $v_{r_{N}}=0$ or capture \\
\hline DMOC $1-1$ & 200 & 500 & Yes & $v_{r_{N}}=0$ \\
DMOC 1-2 & 200 & {$[100: 5000]$} & Yes & Capture \\
DMOC 2-1 & 200 & 249 & Yes & $v_{r_{N}}=0$ \\
DMOC 2-2 & 200 & {$[400: 1000]$} & Yes & Capture \\
DMOC 3-1 & 200 & 685 & Yes & $v_{r_{N}}=0$ \\
DMOC 3-2 & 200 & {$[500: 1000]$} & Yes & Capture \\
DMOC 4-1 & 200 & 267 & Yes & $v_{r_{N}}=0$ \\
DMOC $4-2$ & 200 & {$[500: 1000]$} & Yes & capture \\
\hline \hline
\end{tabular}

$$
J_{d}\left(u_{d}\right)=\sum_{k} h_{k}\left\|\left(u_{k}\right)_{d}\right\|^{2}
$$

where $\left(u_{k}\right)_{d}=\left\{\left(u_{x, k}, u_{y, k}\right)\right\}_{k=0}^{N-1}$ is a vector of length $2 N$, corresponding to $N+1$ total discretization points (nodes), and $\|\cdot\|$ denotes the 2 norm. The total $\Delta V$ applied throughout the trajectory, based on the control forces computed with DMOC, is given by

$$
\Delta V_{\text {traj }}=\alpha_{V} \sum_{k} h_{k}\left\|\left(u_{k}\right)_{d}\right\|
$$

where $\alpha_{V}$ scales the velocity to units of meters per second. Note that $\Delta V_{E}$ and $\Delta V_{M}$ are not included in the cost function for several reasons. The expression for $\Delta V$ is not differentiable, leading to numerical problems and convergence issues. Furthermore, the magnitudes of $\Delta V$ are much larger than the magnitude of the control effort at any given node, so $\Delta V_{E}$ and $\Delta V_{M}$ would dominate the optimization if included. For the optimization, an appropriate weighted sum would have to be chosen, where different choices of weights would lead to different optimal solutions. In addition, control effort and $\Delta V$ are fundamentally different quantities, and thus should not be summed up in one single objective function. To consider different objective functions simultaneously requires multi-objective optimization (see, e.g., [27]), which enables the computation of an entire set of Pareto-optimal solutions being optimal compromises between different objective functions. A multi-objective optimization of this problem is out of the scope of this work, but can be considered in the future.

The optimization process is performed using SNOPT. The primary advantage of SNOPT is its ability to handle large, sparse, nonlinear programing problems. This problem is large, requiring more than 1000 nodes for basic results. A trajectory with $N+1$ nodes results in $2 N+2$ constraints and $8 N^{2}+12 N+4$ constraint derivatives. Obviously, this is a huge endeavor in terms of memory. Fortuitously, this problem is very sparse; most of the constraint derivatives are zero. SNOPTallows the user to provide analytical expressions for the derivatives, and to store these as sparse matrices, saving a great deal of memory and speeding up the process.

\section{B. Optimization Results}

The optimization results for methods 1 and 2 are summarized in Table 3 , and the initial guess and optimal trajectories are shown in Figs. 10 and 11. Each optimal trajectory is different and quite similar to its initial guess, demonstrating the local nature of DMOC and the sensitivity of the results to the initial guess. Most important, $\Delta V_{\text {traj }}$ is reduced to zero. With the correct initial condition, it travels to the moon using no fuel. Also, both $\Delta V_{E}$ and $\Delta V_{M}$ are reduced because the radial velocity constraint forces the initial and final (if used) node of the trajectory to be tangent to a circular orbit at the same radius. These impulsive $\Delta V$ are included for completeness, but are not explicitly included in the optimization or objective function.

Figures 10a and 10d show the optimal trajectories and initial guesses for IG 1 and $\overline{\mathrm{IG}} 2$; Figs. $10 \mathrm{~b}$ and $10 \mathrm{e}$ display the trajectory integrated beyond the final node with zero $\Delta V$ in the EM rotating frame. As shown in Fig. 10b, DMOC 1-1 is not ballistically captured, even though the initial guess had capture because the final distance at the moon is reduced from 2614 to just $500 \mathrm{~km}$ without enforcing capture. For DMOC 1-2, capture is maintained without any additional $\Delta V$. Figure $10 \mathrm{c}$ shows the circular orbit at the moon when $\Delta V_{M}$ is applied at the final node of the optimal trajectory. Notice that DMOC 1-2 is ballistically captured before the final node, so the trajectory loops around the moon once before entering the permanent circular orbit. As shown in Figs. 10e and 10f, continuation of the trajectory shows that ballistic capture is naturally maintained for both DMOC 2-1 and 2-2, and the application of $\Delta V_{M}$ at the final node leads to a permanent circular orbit.

Figure 11 shows similar results for the optimal trajectories generated for method 2 initial guesses. Unlike the results shown in Fig. 10, the different constraints have less of an impact on the optimal results. DMOC 3-1 and 3-2 are very similar, as are DMOC 4-1 and 4-2. All four optimal trajectories naturally maintain ballistic capture at the moon, as shown in Figs. $11 \mathrm{~b}$ and $11 \mathrm{e}$. Despite unreasonably high values for $\Delta V_{E}$ and $\Delta V_{M}$ in the initial guesses, the solutions converge to reasonable values of approximately $3.2 \mathrm{~km} / \mathrm{s}$ for $\Delta V_{E}$ and 5-6 km/s for $\Delta V_{M}$. As before, $\Delta V_{M}$ applied at the final node injects the spacecraft into a permanent circular orbit.

\section{Low Thrust}

The lack of control throughout the trajectory means that this is not actually optimal control. DMOC essentially adjusts the boundary conditions until a free transfer is found. These results are interesting nonetheless. Also, as shown in the preceding sections, an impulsive maneuver is still required for permanent capture at the moon. Therefore, this section explores the use of low-thrust propulsion to spiral into an elliptical orbit at the moon.

\section{A. Formulation of Initial Guess}

Because this problem focuses on the trajectory behavior near the moon, it is natural to consider the dynamics of the planar four-body problem in the Earth-moon rotating frame. Initially, attempts were made using the same four-body model in sun-Earth rotating coordinates as for the impulsive thrust case, but it quickly proved too difficult to generate the desired initial guess capturing the desired sensitive behavior near the moon. The low-thrust equations of motion describe the motion of the spacecraft controlled with lowthrust parameter given by $T=\left(T_{x}, T_{y}\right)$, where $T_{x}$ is the thrust applied in the $x$ direction and $T_{y}$ is the thrust applied in the $y$ direction. The thrust $T$ is limited by $T_{\max }$ to guarantee that the thrust is achievable with low-thrust propulsion. The model also takes into account the mass consumption as a function of the applied thrust. The equations of motion in Earth-moon rotating coordinates are $[\underline{5}, \underline{36}]$

$$
\ddot{x}-2 \dot{y}=\frac{\partial \Omega}{\partial x}+\frac{T_{x}}{m}
$$

Table 3 Details of optimization

\begin{tabular}{lrrrrrrrr}
\hline \hline & $1-1$ & $1-2$ & $2-1$ & $2-2$ & $3-1$ & $3-2$ & $4-1$ & $4-2$ \\
\hline Flight time, days & 168 & 168 & 161 & 161 & 98 & 98 & 95 & 95 \\
Total $\Delta V, \mathrm{~m} / \mathrm{s}$ & 3844 & 3803 & 3853 & 4025 & 3764 & 3790 & 3824 & 3787 \\
$\left.\Delta V_{E}, \mathrm{~m} / \mathrm{s}\right)$ & 3240 & 3240 & 3240 & 3240 & 3212 & 3212 & 3212 & 3212 \\
$\Delta V_{M}, \mathrm{~m} / \mathrm{s}$ & 604 & 563 & 613 & 785 & 552 & 578 & 612 & 575 \\
$\Delta V_{\text {traj }}, \mathrm{m} / \mathrm{s}$ & 0 & 0 & 0 & 0 & 0 & 0 & 0 & 0 \\
$d_{\text {EO }}, \mathrm{km}$ & 200 & 200 & 200 & 200 & 200 & 200 & 200 & 200 \\
$d_{\mathrm{MO}}, \mathrm{km}$ & 500 & 5000 & 249 & 931 & 685 & 500 & 267 & 500 \\
\hline \hline
\end{tabular}




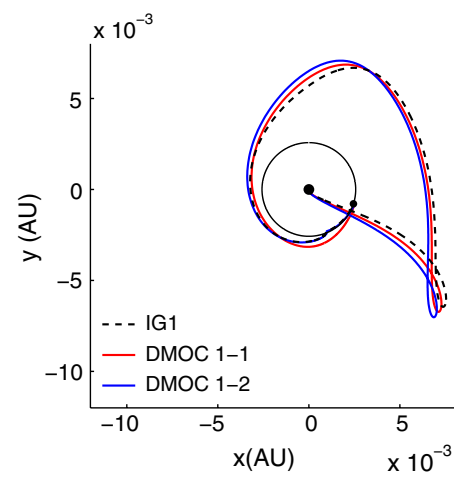

a) DMOC 1

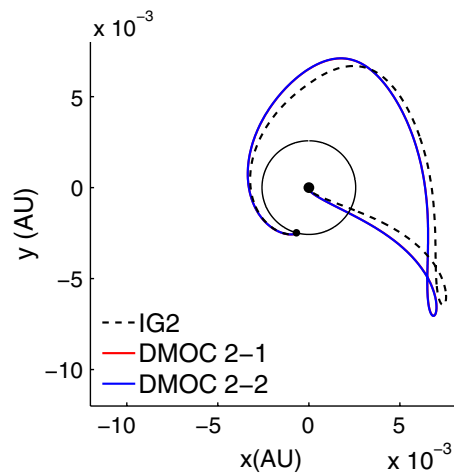

d) DMOC 2

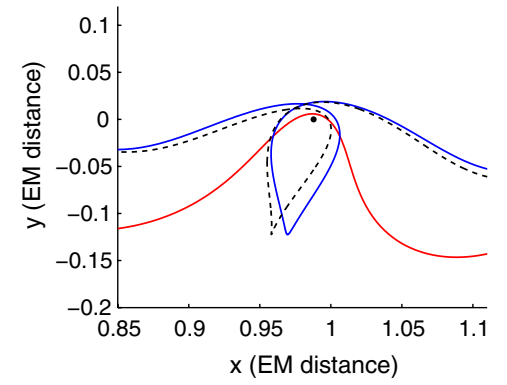

b) $\Delta V_{N}=\mathbf{0}$

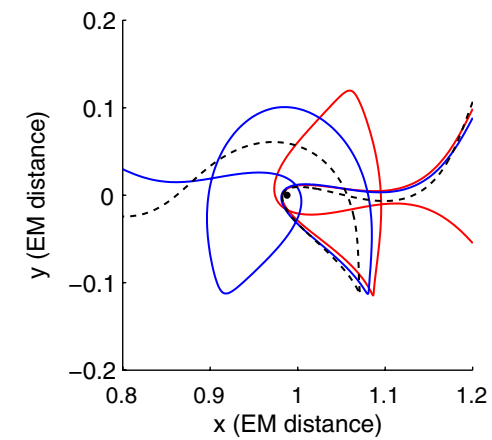

e) $\Delta V_{N}=0$

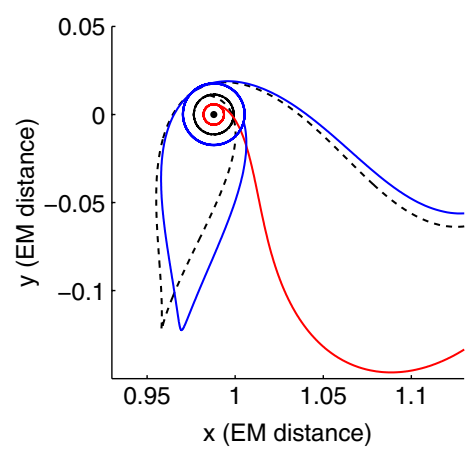

c) $\Delta V_{M} \rightarrow$ circular orbit

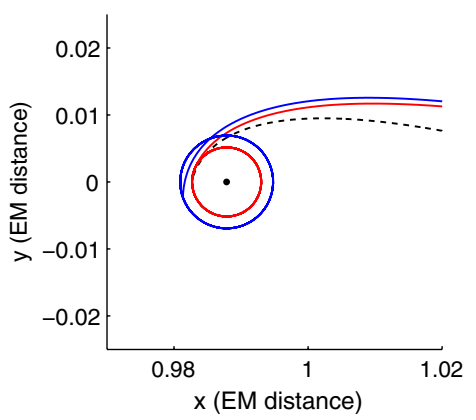

f) $\Delta V_{M} \rightarrow$ circular orbit

Fig. 10 DMOC results generated using different constraints for method 1. Optimal trajectories in SE rotating frame for a) IG1 and d) IG2. Natural capture behavior at the moon in EM rotating frame for b) IG1 and e) IG2. Circular orbit at the moon facilitated by $\Delta V_{M}$ applied at final node of optimal trajectory for c) IG1 and f) IG2.

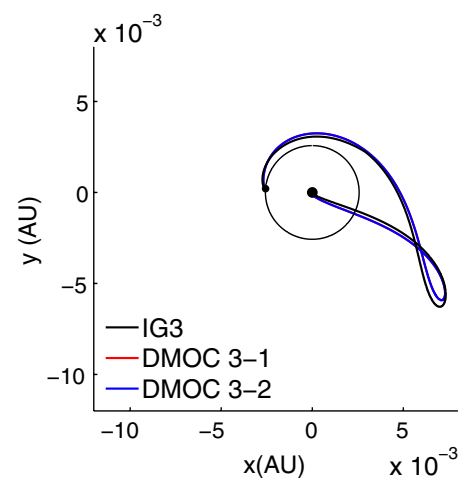

a) DMOC 3

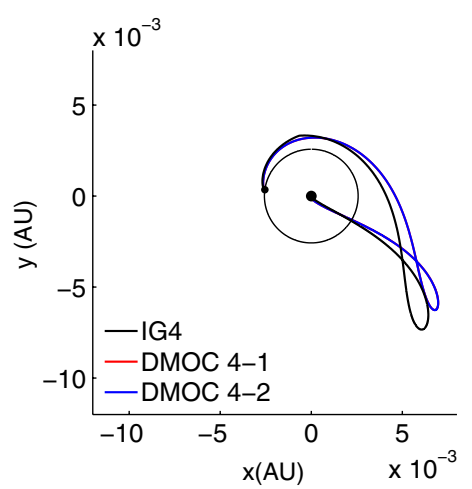

d) $\mathrm{DMOC} 4$

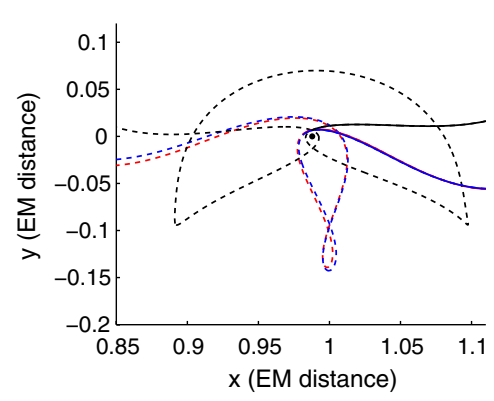

b) $\Delta \boldsymbol{V}_{N}=\mathbf{0}$

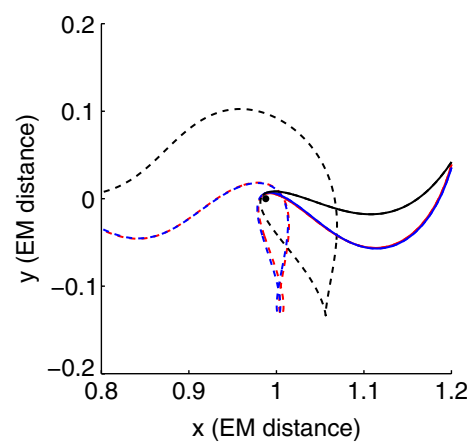

e) $\Delta V_{N}=0$

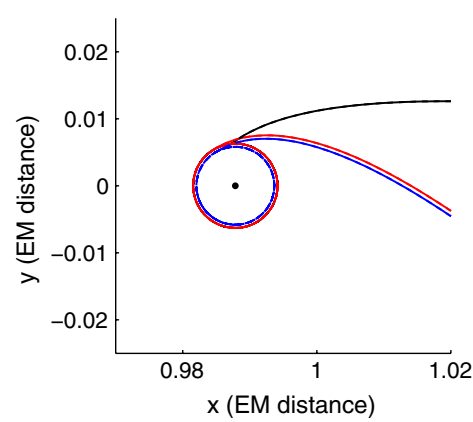

c) $\Delta V_{M} \rightarrow$ circular orbit

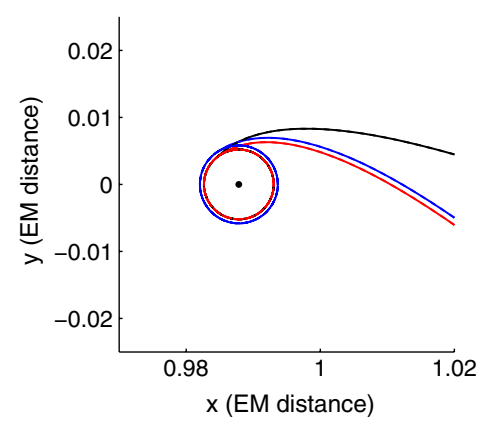

f) $\Delta V_{M} \rightarrow$ circular orbit

Fig. 11 DMOC results generated using different constraints for method 2. Optimal trajectories in SE rotating frame for a) IG3 and d) IG4. Natural capture behavior at the moon in EM rotating frame for b) IG3 and e) IG4. Circular orbit at the moon facilitated by $\Delta V_{M}$ applied at final node of optimal trajectory for c) IG3 and f) IG4. 


$$
\begin{gathered}
\ddot{y}+2 \dot{x}=\frac{\partial \Omega}{\partial y}+\frac{T_{y}}{m} \\
\dot{m}=-\frac{T}{I_{\mathrm{sp}}^{\mathrm{LT}} g_{0}}
\end{gathered}
$$

where

$$
\begin{gathered}
0 \leq T=\sqrt{T_{x}^{2}+T_{y}^{2}} \leq T_{\max } \\
\Omega=\frac{x^{2}+y^{2}}{2}+\frac{m_{E}}{\sqrt{(x+\mu)^{2}+y^{2}}}+\frac{m_{M}}{\sqrt{(x+(1-\mu))^{2}+y^{2}}} \\
+\frac{m_{S}}{\sqrt{\left(x-x_{S}\right)^{2}+\left(y-y_{S}\right)^{2}}}-\frac{m_{S}}{a_{S}^{3}}\left(x_{S}+y_{S}\right)
\end{gathered}
$$

and $m_{S}, m_{E}$, and $m_{M}$ are the normalized mass of the sun, Earth, and moon, respectively, given by

$$
\begin{gathered}
m_{E}=1-\mu \\
m_{M}=\mu \\
m_{S}=\frac{M_{S}}{M_{M}+M_{E}}=3.2890 \times 10^{5}
\end{gathered}
$$

and

$$
\mu=\frac{M_{M}}{M_{E}+M_{M}}=0.01215
$$

As before, $M_{i}, i=E, M, S$, denotes the body's mass in kilograms, and $x_{S}$ and $y_{S}$ represent the $x$ and $y$ positions of the sun as a function of time given by

$$
\begin{gathered}
\theta_{S}=-\omega_{S} t+\theta_{S 0} \\
x_{S}=a_{S} \cos \theta_{S} \\
y_{S}=a_{S} \sin \theta_{S}
\end{gathered}
$$

where $t$ is time, $\theta_{S 0}$ is the initial angle of the sun with respect to the $x$ axis in the Earth-moon rotating frame, $a_{S}=3.8881 \times 10^{2}$ is the normalized radius of the sun's circular orbit, and $\omega_{S}=0.9251$ is the normalized rotation rate of the sun. Note that $I_{\mathrm{sp}}^{\mathrm{LT}}=3000 \mathrm{~s}$ is the specific impulse of the low-thrust (LT) thruster, $g_{0}=9.81 \mathrm{~m} / \mathrm{s}^{2}$ is the acceleration due to gravity at sea level, and $T_{\max }$ is the maximum allowable thrust; in this case, $0.5 \mathrm{~N}$ is used. As before, all of these values are normalized and nondimensional. As for the four-body model with impulsive control, this low-thrust four-body model is also dynamically incoherent, but it is useful nonetheless.

The performance of a low-thrust trajectory is measured based on the fuel mass consumption $m_{p}$ and the mass fraction $\frac{m_{p}}{m_{0}}$, where

$$
m_{p}=m_{0}-m_{F}
$$

is the difference of the initial and final mass. To determine the mass fraction for an impulsive $\Delta V$,

$$
\frac{m_{p}}{m_{0}}=1-\exp \left(-\frac{\sum_{i} \Delta V_{i}}{I_{\mathrm{sp}}^{\mathrm{HT}} g_{0}}\right)
$$

where $I_{\mathrm{sp}}^{\mathrm{HT}}=300 \mathrm{~s}$ is the specific impulse of an impulsive thruster, where HT denotes high thrust, and $\Delta V_{i}$ are the impulsive $\Delta V$.

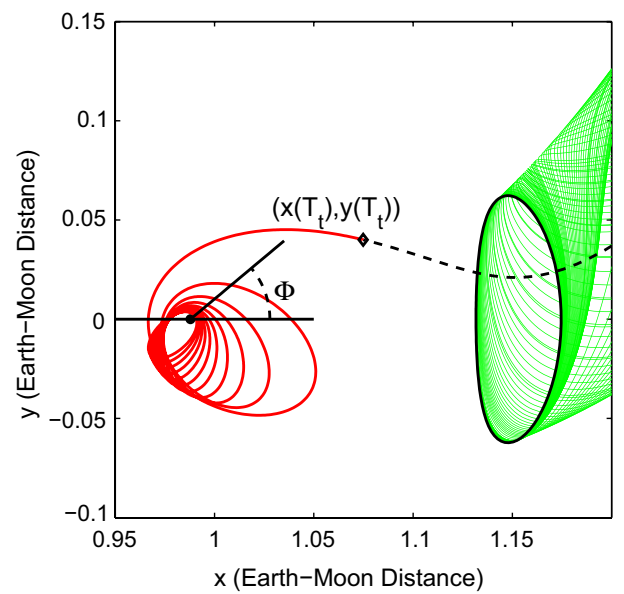

Fig. 12 Low-thrust spiral with variable parameters $T_{t}$ and $\phi . T_{t}$ marks the transition between no thrust and low thrust, and $\phi$ is the angle of the semimajor axis of the elliptical orbit at the moon with respect to the Earth-moon $x$ axis.

The desired optimal trajectory ends in an elliptical orbit at the moon; therefore, that elliptical orbit is used as a starting point for formulation of the initial guess. First, the conditions $(x, y, \dot{x}, \dot{y})$ of an elliptical orbit with eccentricity $e=0.65$ and desired distance from the moon are integrated backward applying the maximum value of thrust. The goal is to generate a trajectory that spirals out from the initial elliptical orbit, flows through the periodic orbit at $L_{2}$, and travels through the stable Earth-moon manifold toward its intersection with the unstable sun-Earth manifold, the patch point. Two variables may be manually adjusted to achieve this goal: $T_{t}$, the time at which the low-thrust is turned off, and $\Phi$, the phase angle of the ellipse with respect to the Earth-moon rotating frame's $x$ axis (shown in Fig. 12). Experimenting with different values for these variables, the desired trajectory emerges. Figures $13 \mathrm{a}$ and $13 \mathrm{c}$ show the trajectory in the Earth-moon and sun-Earth rotating frames, respectively, for one set of $\left(T_{t}, \Phi\right)$ values, whereas Figs. $13 \mathrm{~b}$ and $13 \mathrm{~d}$ shows the trajectory for a different set of $\left(T_{t}, \Phi\right)$ values. Note that the low-thrust spiral will not be included in the initial guess for optimization; instead, it is fixed.

For this low-thrust trajectory, time $t=0$ is set at the desired final point of the trajectory near the moon, and time flows backward. This differs from the original case when designing an initial guess in the sun-Earth rotating frame using method 1 , for which time $t=0$ occurs at the patch point. To ensure the same manifold intersection as before, $\theta_{S_{0}}$, the initial angle of the sun with respect to the Earth-moon $x$ axis, is chosen such that $\theta_{M}$ at the patch point equals $\theta_{M_{0}}$ from method 1 . This is achieved using an iterative process in which a guess is posed for $\theta_{S_{0}}$, the trajectory is integrated backward to the patch point, $\theta_{M}$ is computed, and $\theta_{S_{0}}$ is refined. This process continues until $\theta_{M}$ (patch point $)-\theta_{M_{0}(\text { method } 1)}<$ tolerance.

When the patch point is reached, an impulsive $\Delta V$ is applied and integration continues until the trajectory reaches the Earth. This $\Delta V$ may be adjusted until the desired radius at the Earth is reached. The full trajectory is shown in Figs. $13 \mathrm{c}$ and $13 \mathrm{~d}$ in sun-Earth rotating coordinates.

If the initial conditions at the moon are integrated forward with no thrust, the desired elliptical orbit at the moon results. Recall that the majority of the trajectory, except for the small portion near the moon and at the patch point, is achieved using zero thrust. For optimization purposes, the trajectory is reorganized such that it begins at the Earth and ends near the moon. The initial guess ends at the last node before low thrust is applied, one node before $T_{t}$, excluding the low-thrust spiral toward the moon from the optimization.

Two initial guesses are tested. Both trajectories begin approximately $167 \mathrm{~km}$ from the surface of the Earth. IG LT1 requires an impulsive $\Delta V=68 \mathrm{~m} / \mathrm{s}$ at the patch point and, with lowthrust spiral, ends in an elliptical orbit with $d_{p}=1000 \mathrm{~km}$. The mass fraction for the low-thrust portion of the trajectory is 0.0158 , and 


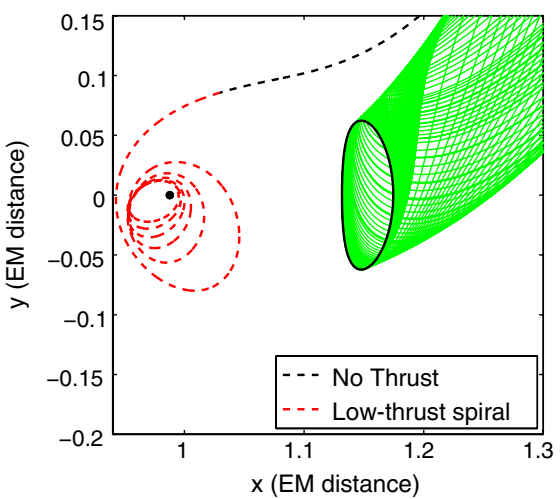

a)Low-thrust spiral, IG LT1

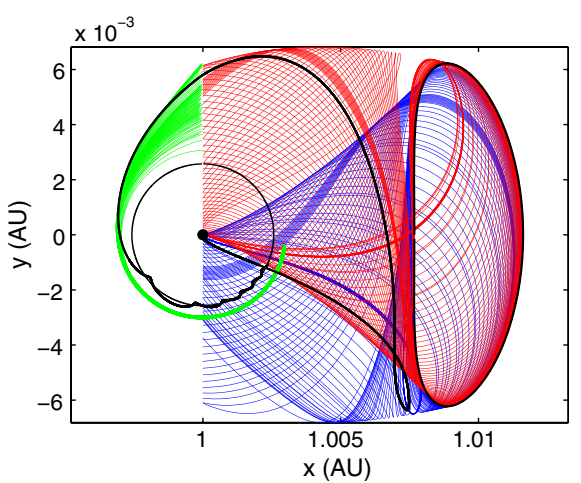

c) Full trajectory (SE rotating frame), IG LT1

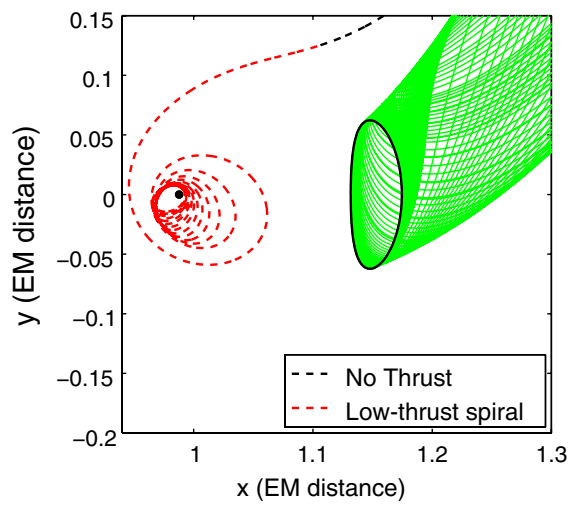

b) Low-thrust spiral, IG LT2

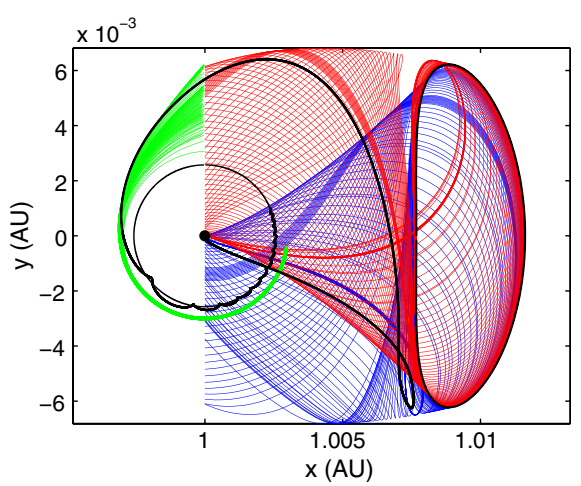

d) Full trajectory (SE rotating frame), IG LT2

Fig. 13 Initial guess trajectories with low thrust are achieved by integrating backward from desired elliptical orbit at the moon. Maximum thrust is applied in the direction opposite the velocity until reaching time $T_{t}$, and then integration continues without thrust for a) IG LT1 and b) IG LT2. The full trajectory, including a small impulsive $\Delta V$ at the patch point, ends $167 \mathrm{~km}$ from the Earth in the sun-Earth rotating frame for c) IG LT1 and d) IG LT2.

according to Eq. (49), the mass fraction for the impulsive $\Delta V$ is 0.0230 , giving a total mass fraction of 0.0388 . The second initial guess, IG LT2, requires an impulsive $\Delta V=33 \mathrm{~m} / \mathrm{s}$ at the patch point and ends in a $100 \mathrm{~km}$ elliptical orbit at the moon. The total mass fraction is 0.0356 with 0.0243 for the low-thrust spiral and 0.0113 for the impulsive $\Delta V$.

\section{B. Low-Thrust Optimization}

The optimization process differs from that of method 1 and method 2. In particular, the optimized solution should be achievable using only low thrust. As before, the primary constraints are the discrete Euler-Lagrange equations, Eq. (25), derived from the Lagrangian

$$
\begin{aligned}
L= & \frac{1}{2}\left(\dot{x}^{2}+\dot{y}^{2}\right)+\frac{1}{2}\left(x^{2}+y^{2}\right)+x \dot{y}-y \dot{x}+\frac{m_{E}}{r_{E}}+\frac{m_{M}}{r_{M}}+\frac{m_{S}}{r_{S}} \\
& -\frac{m_{S}}{a_{S}^{3}}\left(x \cdot x_{S}+y \cdot y_{S}\right)
\end{aligned}
$$

where $r_{E}, r_{M}$, and $r_{S}$ are the distance of the spacecraft from the centers of the Earth, moon, and sun, respectively. Because mass is included in the equations of motion, Eq. (38), the discrete mass at each node $m_{k}$ is an optimization variable, and the mass dynamics are enforced by the constraint equation

$$
m_{k+1}-m_{k}+h_{k}\left(\frac{T_{k}}{I_{\mathrm{sp}}^{\mathrm{LT}} g_{0}}\right)=0
$$

where $T_{k}=\sqrt{T_{x, k}^{2}+T_{y, k}^{2}}$ is the thrust magnitude applied tangentially at each node $k$. There are several options for the definition of $T_{x, k}$ and $T_{y, k}$.

1) $T_{x, k}$ and $T_{y, k}$ are optimization variables that vary within the limits $\left[-T_{\max }, T_{\max }\right]$ subject to the constraint $T_{k}=$ $\sqrt{T_{x, k}^{2}+T_{y, k}^{2}} \leq T_{\max }$.
2) The optimization variable is $\tau_{k}$, and $T_{x, k}$ and $T_{y, k}$ are defined such that the thrust $T_{k}=\left|\tau_{k}\right|$ is applied in the direction parallel to or opposite the velocity vector

$$
\begin{gathered}
T_{x, k}=\tau_{k}\left(\frac{v_{x, k}}{\sqrt{v_{x, k}^{2}+v_{y, k}^{2}}}\right) \\
T_{y, k}=\tau_{k}\left(\frac{v_{y, k}}{\sqrt{v_{y, k}^{2}+v_{y, k}^{2}}}\right) \\
-T_{\max } \leq \tau_{k} \leq T_{\max }
\end{gathered}
$$

There are problems with both of these options that arise in the computation of the derivatives for the constraint Jacobian. Defining thrust according to option 1, consider the derivative of Eq. (1) with respect to $T_{x, k}$,

$$
\begin{aligned}
& \frac{\partial}{\partial T_{x, k}}\left[m_{k+1}-m_{k}+h_{k}\left(\frac{\sqrt{T_{x, k}^{2}+T_{y, k}^{2}}}{I_{\mathrm{sp}}^{\mathrm{LT}} g_{0}}\right)\right] \\
& =\frac{h_{k}}{I_{\mathrm{sp}}^{\mathrm{LT}} g_{0}}\left(\frac{T_{x, k}}{\sqrt{T_{x, k}^{2}+T_{y, k}^{2}}}\right)
\end{aligned}
$$

This derivative is undefined if $T_{x, k}=T_{y, k}=0$, which is allowable. For option 2, since $T_{k}=\left|\tau_{k}\right|$ is not differentiable when $\tau_{k}=0$, the derivative of the mass constraint with respect to $\tau_{k}$ also does not exist everywhere. There are possible tricks in the problem setup that avoid these existence problems, but both strategies display poor convergence results. Ultimately, the best results are achieved when the thrust is defined similar to option 2 , but with a restriction. 
Let $T_{k}$ be the control optimization variable such that the thrust is applied in the direction opposite the velocity (this means that the thrust acts to slow the spacecraft) where

$$
\begin{gathered}
T_{x, k}=T_{k}\left(-\frac{v_{x, k}}{\sqrt{v_{x, k}^{2}+v_{y, k}^{2}}}\right) \\
T_{y, k}=T_{k}\left(-\frac{v_{y, k}}{\sqrt{v_{x, k}^{2}+v_{y, k}^{2}}}\right) \\
0 \leq T_{k} \leq T_{\max }
\end{gathered}
$$

where $v_{x, k}$ and $v_{y, k}$ are the velocity in the $x$ and $y$ directions at node $k$, respectively. The discrete left and right control forces based on this thrust definition are

$$
\begin{aligned}
f_{x, k}^{+} & =f_{x, k}^{-}=\frac{h_{k}}{2} \frac{T_{k}}{\left[\left(m_{k+1}+m_{k}\right) / 2\right]} \\
& \times\left(-\frac{\left[\left(x_{k+1}-x_{k}\right) / h_{k}\right]}{\sqrt{\left[\left(x_{k+1}-x_{k}\right) / h_{k}\right]^{2}+\left[\left(y_{k+1}-y_{k}\right) / h_{k}\right]^{2}}}\right) \\
f_{y, k}^{+} & =f_{y, k}^{-}=\frac{h_{k}}{2} \frac{T_{k}}{\left[\left(m_{k+1}+m_{k}\right) / 2\right]} \\
& \times\left(-\frac{\left[\left(y_{k+1}-y_{k}\right) / h_{k}\right]}{\sqrt{\left[\left(x_{k+1}-x_{k}\right) / h_{k}\right]^{2}+\left[\left(y_{k+1}-y_{k}\right) / h_{k}\right]^{2}}}\right)
\end{aligned}
$$

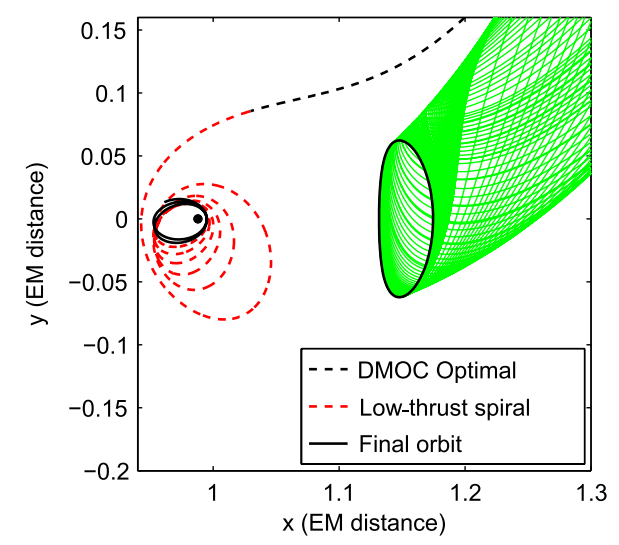

a) Low-thrust spiral in EM rotating frame

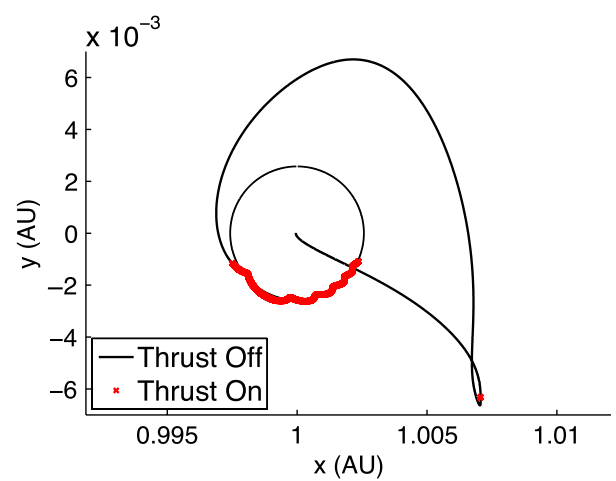

c) SE rotating frame

Fig. 14 DMOC LT1-1: optimal trajectory with low thrust. a) The optimal trajectory is shown near the moon in the Earth-moon rotating frame, with low-thrust spiral and final orbit added. b) Low-thrust spiral and final orbit in moon-centered inertial frame, c) optimized trajectory in sun-Earth rotating frame with low-thrust arcs identified, and d) the optimized trajectory in Earth-centered inertial frame with low-thrust arcs.
In summary, the optimization variables are $x_{k}, y_{k}$, and $m_{k}$ for $k=1, \ldots, N$ and $T_{k}$ for $k=1, \ldots, N-1$, and the constraints that enforce dynamics are

$$
\begin{gathered}
D_{2} L_{d}\left(q_{k-1}, q_{k}\right)+D_{1} L_{d}\left(q_{k}, q_{k+1}\right)+f_{k-1}^{+}+f_{k}^{-}=0 \\
m_{k+1}-m_{k}+h_{k}\left(\frac{T_{k}}{I_{\mathrm{sp}}^{\mathrm{LT}} g_{0}}\right)=0
\end{gathered}
$$

Because the optimized solution should naturally flow into the lowthrust spiral determined for the initial guess, the mass at the end of the optimized trajectory $m_{N}$ is required to be $1000 \mathrm{~kg}$ (the initial mass assumed for the spiral). Additionally, the initial altitude of a circular orbit at the Earth must be $d_{\mathrm{EO}}=167 \mathrm{~km}$ and $v_{r_{0}}=0$. To ensure that the spiraling low-thrust portion remains possible, the position and momentum at the final node must match that of the initial guess.

Two different objective functions are considered. The first objective function aims to minimize the initial mass (since $m_{N}$ is held fixed at $1000 \mathrm{~kg}$ ),

$$
J_{d 1}=m_{0}
$$

The second objective function seeks to minimize the overall control effort, or thrust,

$$
J_{d 2}=\sum_{k} h_{k}\left(\frac{T_{k}}{\left[\left(m_{k+1}+m_{k}\right) / 2\right]}\right)^{2}
$$

These objective functions lead to very similar optimal trajectories converges faster and leads to a thrust profile for which the thrust is turned off for most of the trajectory. Minimizing the control effort, Eq. (64), leads to a continuous thrust profile, with magnitude much less than $T_{\max }$, applied for most of the trajectory. Results for both

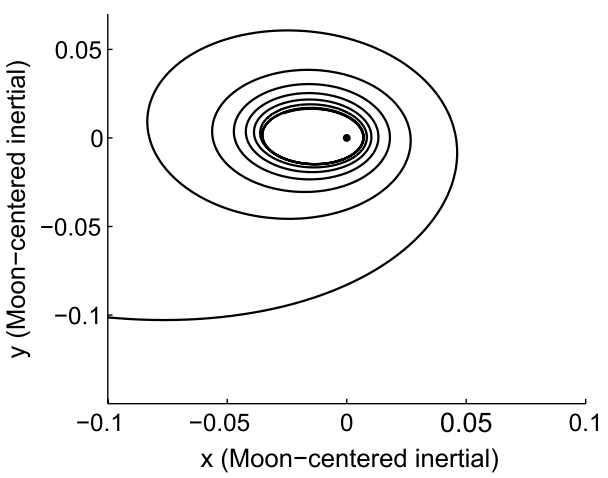

b) Moon-centered inertial frame

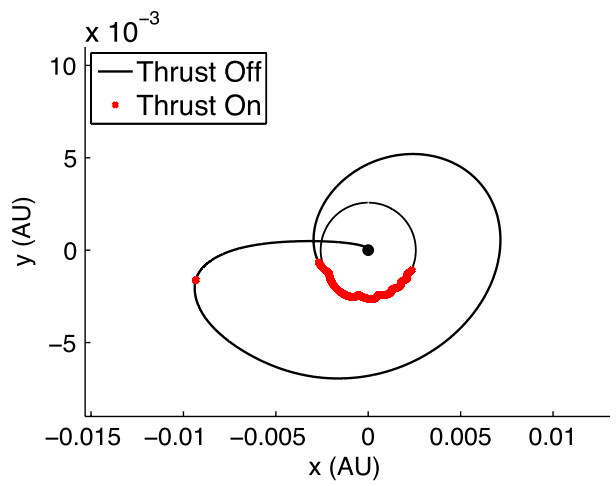

d) Earth-centered inertial frame with different thrust profiles. Optimization with $J_{d 1}$, Eq. (63), 
initial guesses, IG LT1 and IG LT2, are presented for $J_{d 1}$, denoted DMOC LT1-1 and DMOC LT2-1, respectively. Only IG LT1 is optimized with $J_{d 2}$ and because the trajectory results, denoted by DMOC LT1-2, are nearly identical to those of $J_{d 1}$, only the thrust profile and thrust location are shown.

Figure 14 shows the optimization results for DMOC LT1-1. The transition from no thrust to low thrust at the moon is shown in Earthmoon rotating coordinates in Fig. 14a. Figures 14c and 14d show the entire trajectory with the location of thrust arcs identified in sunEarth rotating coordinates and Earth-centered inertial coordinates, respectively. If the final conditions at the end of the low-thrust spiral are integrated with no thrust, the trajectory settles into the desired orbit at the moon, shown in moon-centered inertial coordinates in Fig. 14b. Figure 15 shows the same plots for DMOC LT2-1. Figures $16 \mathrm{a}$ and $\overline{16 \mathrm{~b}}$ show the thrust arcs in sun-Earth rotating

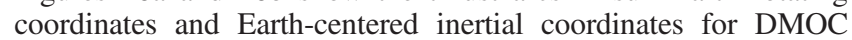
LT1-2.

The thrust profile for DMOC LT1-1 and the low-thrust spiral are shown in Fig. 17a. The majority of the profile consists of zero thrust, with a small maximum thrust arc applied for about $9.5 \mathrm{~h}$ on day 33 . Also, the thruster turns on approximately $7 \mathrm{~h}$ before reaching the beginning of the low-thrust spiral. This optimal trajectory from Earth to elliptical orbit at the moon requires just $16.87 \mathrm{~kg}$ of fuel, giving a total mass fraction of $m_{p} / m_{0}=0.0168$. This thrust profile is nearly bang-bang control; the thrust is either off or at the maximum value.

The entire thrust profile for DMOC LT2-1, including low-thrust spiral, is given in Fig. 17b. This thrust profile contains two smaller, continuous thrust arcs. The first arc begins on day 31, rising continuously to a maximum of $0.1 \mathrm{~N}$ before decreasing back to zero by day 32. The second thrust arc begins on day 146, reaches a maximum of $0.29 \mathrm{~N}$, and ends approximately 1 day later. The mass fraction for the entire trajectory is $m_{p} / m_{0}=0.0249$ and uses just $24.9 \mathrm{~kg}$ of fuel. As shown by the similarity of the optimal trajectories in Figs. 14 and 15 , the trajectory itself is relatively insensitive to the starting point of the low-thrust spiral. Any differences, though small, are in the location and magnitude of the thrust.

The thrust profile for DMOC LT1-2, excluding the low-thrust spiral, is shown in Fig. 18. The thrust is continuous, except for the drop off to zero at the final node. The thrust turns on approximately 1 day into the trajectory. The thrust steadily increases to a maximum of $0.0065 \mathrm{~N}$ on day 34 and then decreases back to zero on day 79 . The thrust is then off until day 113 when it begins to increase to a maximum of $0.016 \mathrm{~N}$, decreases slightly, and increases again to the global maximum of $0.0371 \mathrm{~N}$ on day 153 before shutting off. This trajectory requires $17.9 \mathrm{~kg}$ of fuel and its total mass fraction is $m_{p} / m_{0}=0.0179$. Notice the difference in scale between Figs. 17 and 18 ; the thrust profile resulting from $J_{d 2}$ leads to thrust magnitudes more than an order of magnitude less than those generated by $J_{d 1}$.

Table 4 summarizes the optimization results. The impulsive $\Delta V$ at the patch point for the initial guess trajectories is included. However, this metric is meaningless for the low-thrust optimal solutions and is not provided for those results. Note that the mass fraction $m_{p} / m_{0}$ includes the optimized thrust from DMOC as well as the low-thrust spiral. Of the three optimal results, DMOC LT1-1 results in the smallest mass fraction and takes 4 days less than DMOC LT2-1. Figure 19 displays the mass throughout the trajectory, including the fuel mass consumed during low-thrust spiral at the end. DMOC LT12 requires the highest starting mass. DMOC LT2-1 requires the smallest initial mass, but the low-thrust spiral burns more fuel than the other low-thrust spirals, resulting in the highest mass fraction.

\section{Analysis and Comparison}

Table $\underline{5}$ displays several trajectory results from literature to compare with the DMOC solutions. The first four trajectories are presented by Belbruno and Miller [1] where WSB, BP, H, and BE stand for weak stability boundary, biparabolic, biellipctic, and
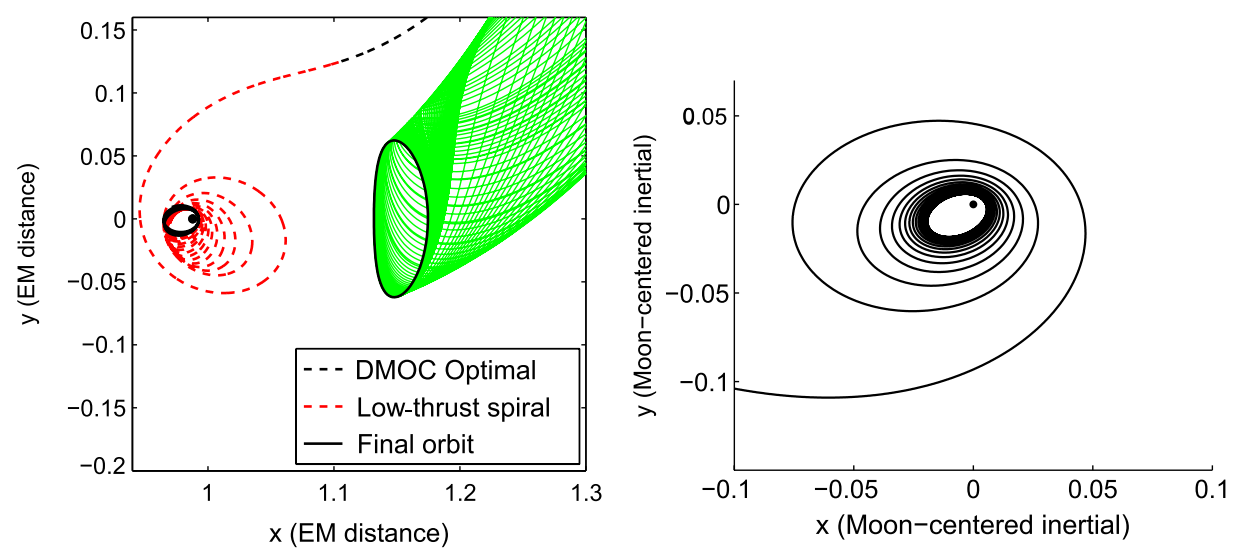

a) Low-thrust spiral in EM rotating frame

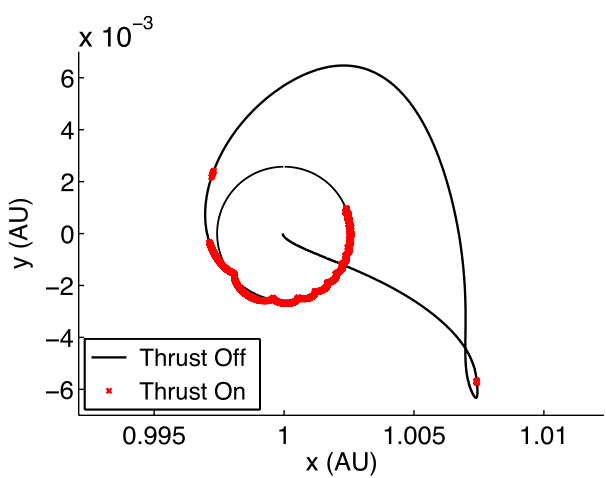

b) Moon-centered inertial frame

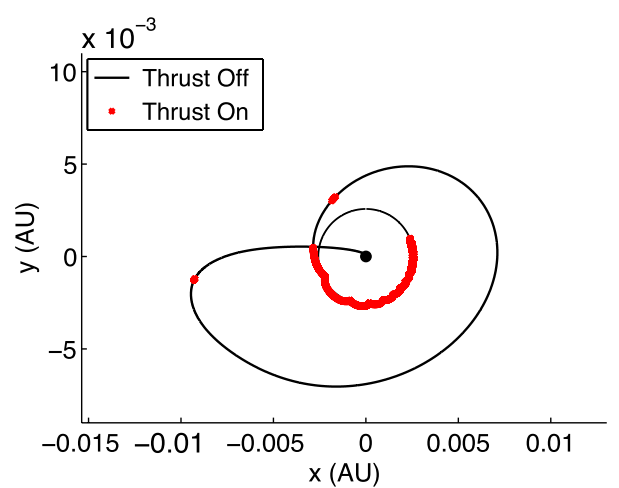

d) Earth-centered inertial frame

c) SE rotating frame

Fig. 15 DMOC LT2-1: optimal trajectory with low thrust. a) The optimal trajectory is shown near the moon in the Earth-moon rotating frame, with low-thrust spiral and final orbit added. b) Low-thrust spiral and final orbit in moon-centered inertial frame, c) optimized trajectory in sun-Earth rotating frame with low-thrust arcs identified, and d) the optimized trajectory in Earth-centered inertial frame with low-thrust arcs. 


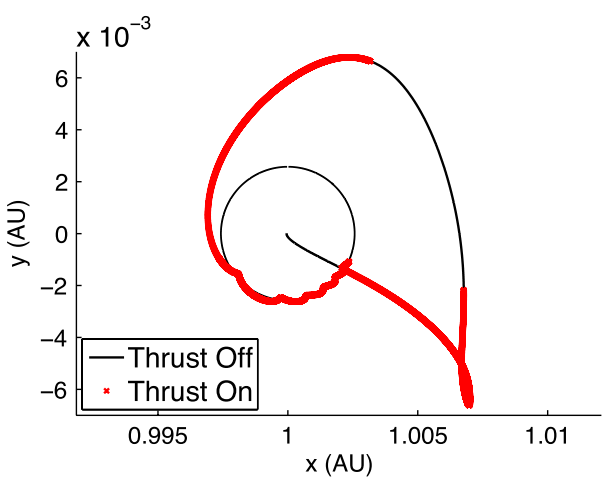

a) $\mathrm{SE}$ rotating frame

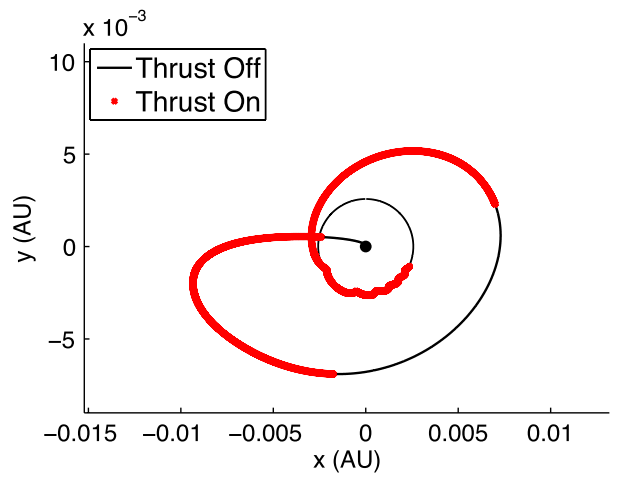

b) Earth-centered inertial frame

Fig. 16 DMOC LT1-2: optimal trajectory with low thrust, generated with cost function $J_{d 2}$. a) Optimized trajectory in sun-Earth rotating frame with low-thrust arcs identified, and b) the optimized trajectory in Earth-centered inertial frame with low-thrust arcs.

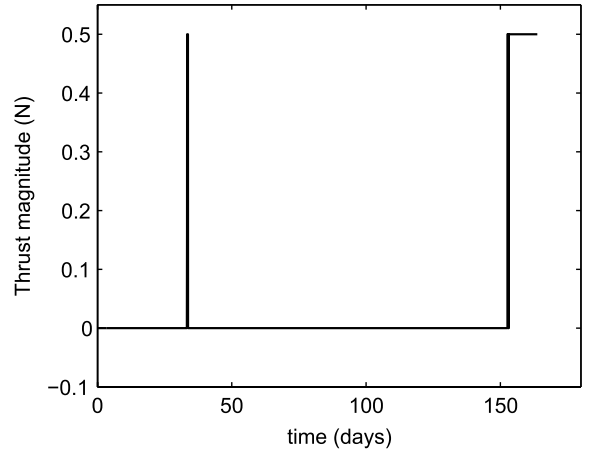

a) DMOC LT1-1 thrust profile

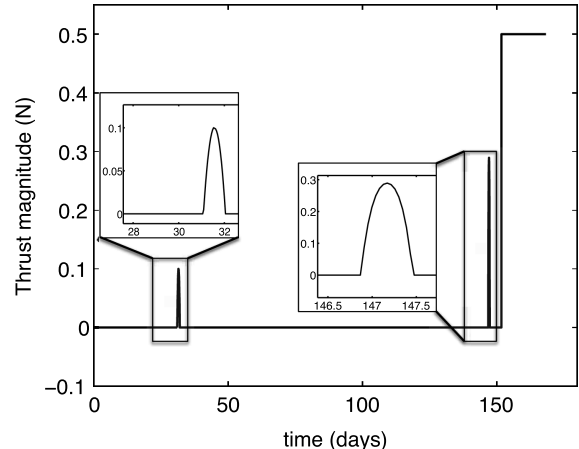

b) DMOC LT2-1 thrust profle

Fig. 17 Thrust profile: magnitude of thrust throughout the trajectory for a) DMOC LT1-1 and b) DMOC LT2-1.

Hohmann transfers, respectively. All of these trajectories begin in a $167 \mathrm{~km}$ circular Earth orbit and end in a $100 \mathrm{~km}$ circular orbit at the moon. The WSB trajectory is very similar to that of Shoot the Moon and produces a low-energy transfer trajectory that connects the weak stability boundaries (closely related to invariant manifolds) of the sun-Earth and Earth-moon systems. Additionally, the Shoot the Moon trajectory presented by Koon et al. [4] is represented by SM. This trajectory begins in a $200 \mathrm{~km}$ circular orbit, just like the DMOC solutions, and ends in ballistic capture. The final orbit and $\Delta V_{M}$ required to circularize the orbit at the moon are not provided for SM.

For each trajectory, certain metrics are compared, as done in Belbruno and Miller [1]. First, it is assumed that the launch vehicle provides an injection $\Delta V$ equal to that necessary for a Hohmann transfer from that particular altitude orbit. The first four trajectories begin in a $167 \mathrm{~km}$ orbit, requiring $\Delta V_{H}=3.143 \mathrm{~km} / \mathrm{s}$ to leave Earth orbit using a Hohmann transfer. Any additional $\Delta V$ required for injection, denoted $\Delta V_{E}-\Delta V_{H}$, must be included in the mission

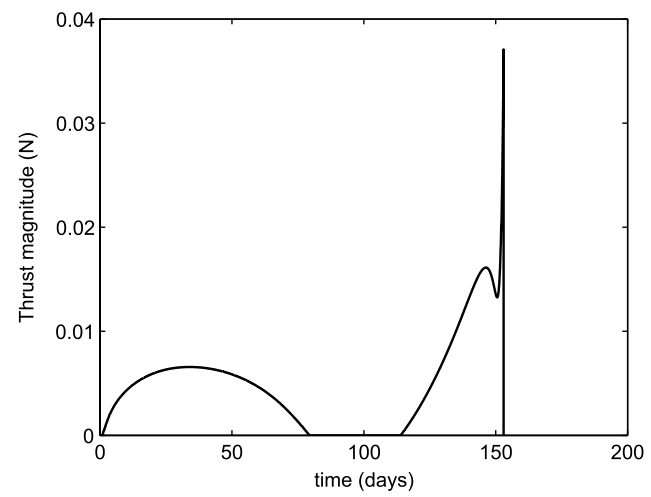

Fig. 18 Thrust profile: magnitude of low thrust throughout trajectory for DMOC LT1-2.
$\Delta V$. The DMOC and $\mathrm{SM}$ results begin in a $200 \mathrm{~km}$ orbit, requiring $\Delta V_{H}=3.149 \mathrm{~km} / \mathrm{s}$ for the Hohmann transfer. Next, midcourse $\Delta V$ are compared, as denoted by $\Delta V_{\text {traj. }}$. For the WSB, BP, H, and BE trajectories, it is assumed that the spacecraft is first captured at the moon in an elliptical orbit with eccentricity $e=0.95$, and periapsis radius $r_{p}=r_{M}+100 \mathrm{~km}$, where $r_{M}$ is the radius of the moon. The $\Delta V$ necessary to enter this elliptical capture orbit is denoted by $\Delta V_{C}$,

$$
\Delta V_{C}=\sqrt{V_{\infty}^{2}+\frac{2 m_{M}}{r_{p}}}-\sqrt{\frac{2 m_{M} r_{a}}{\left(r_{a}+r_{p}\right) r_{p}}}
$$

where $r_{a}$ is the radius of the orbit at apoapsis and

$$
V_{\infty}=\sqrt{m_{M}\left(\frac{2}{r_{M_{H}}}-\frac{1}{a_{H}}\right)}-\sqrt{\frac{m_{M}}{r_{M_{H}}}}
$$

where $r_{M_{H}}$ and $a_{H}$ are the periapsis and semimajor axis of the Hohmann transfer ellipse, respectively. Finally, $\Delta V_{M}$ is necessary to circularize the orbit at the periapsis. The DMOC solutions do not include $\Delta V_{C}$; they are circularized directly from the final node. The total measure of $\Delta V$ performance is given by $\sum \Delta V=$ $\Delta V_{E}-\Delta V_{H}+\Delta V_{\text {traj }}+\Delta V_{C}+\Delta V_{M}$. All numbers in the table are given in kilometers per second.

As shown by the percent change from the corresponding Hohmann transfer, the DMOC results are at most $19 \%$ better than the Hohmann transfer (DMOC 3-1). Two of the DMOC results, DMOC 1-2 and 2-2, are actually worse than the Hohmann transfer, requiring $7 \%$ and $9 \%$ more fuel, respectively. Both of these results lack the constraint requiring the radial velocity at the final node to be zero, demonstrating the importance of that constraint. The other DMOC results are competitive with those from the literature. An emphasis on 
Table 4 Details of optimization

\begin{tabular}{lccccc}
\hline \hline & LT IG1 & DMOC LT1-1 & DMOC LT1-2 & LT IG2 & DMOC LT2-1 \\
\hline Flight time & 164 & 164 & 164 & 168 & 168 \\
$\Delta V_{E}, \mathrm{~m} / \mathrm{s}$ & 3190 & 3190 & 3190 & 3189 & 3189 \\
$\Delta V_{M}, \mathrm{~m} / \mathrm{s}$ & 381 & 381 & 381 & 465 & 465 \\
$\Delta V_{\text {traj }}, \mathrm{m} / \mathrm{s}$ & 68 & - & 167 & 33 & -167.4 \\
Earth orbit altitude, $\mathrm{km}$ & 167.8 & 167 & 1000 & 100 & 167 \\
Moon orbit altitude, $\mathrm{km}$ & 1000 & 1000 & 0.0179 & 0.0356 & 0.0249 \\
$m_{p} / m_{0}$ & 0.0388 & 0.0168 & & & \\
\hline \hline
\end{tabular}

Table 5 Comparison of trajectories with impulsive thrust

\begin{tabular}{lclcccr}
\hline \hline Type & $\Delta V_{E}-\Delta V_{H}$ & $\Delta V_{\text {traj }}$ & $\Delta V_{C}$ & $\Delta V_{M}$ & $\sum \Delta V$ & \% change from $H$ \\
\hline WSB & 0.018 & 0.029 & 0 & 0.648 & 0.695 & -18 \\
BP & 0.089 & 0 & 0.073 & 0.648 & 0.810 & -4 \\
H & 0.000 & 0 & 0.2 & 0.648 & 0.848 & 0 \\
BE & 0.018 & 0.287 & 0.052 & 0.648 & 1.005 & 19 \\
DMOC 1-1 & 0.090 & 0 & - & 0.604 & 0.694 & -11 \\
DMOC 1-2 & 0.091 & 0 & - & 0.562 & 0.653 & 7 \\
DMOC 2-1 & 0.091 & 0 & - & 0.613 & 0.704 & -13 \\
DMOC 2-2 & 0.091 & 0 & - & 0.785 & 0.876 & -19 \\
DMOC 3-1 & 0.062 & 0 & - & 0.552 & 0.614 & -17 \\
DMOC 3-2 & 0.062 & 0 & - & 0.578 & 0.640 & -16 \\
DMOC 4-1 & 0.063 & 0 & - & 0.612 & 0.675 & -18 \\
DMOC 4-2 & 0.063 & 0 & - & 0.575 & 0.638 & - \\
SM & 0.062 & 0.034 & - & - & - & \\
\hline \hline
\end{tabular}

Table 6 Comparison of trajectories with low thrust

\begin{tabular}{lcrccc}
\hline \hline Type & $\Delta V_{E}, \mathrm{~m} / \mathrm{s}$ & $r_{p}, \mathrm{~km}$ & $e$ & $m_{p} / m_{0}$ & Flight time, days \\
\hline LT ref 1 & 3195 & 1000 & 0.65 & 0.031 & 236 \\
LT ref 2 & 3203 & 100 & 0.65 & 0.061 & 228 \\
DMOC LT1-1 & 3190 & 1000 & 0.65 & 0.0168 & 164 \\
DMOC LT2-1 & 3189 & 100 & 0.65 & 0.0249 & 178 \\
DMOC LT1-2 & 3190 & 1000 & 0.65 & 0.0179 & 164 \\
\hline \hline
\end{tabular}

$\Delta V_{E}$ and $\Delta V_{M}$ for the initial guess or in the optimization may lead to even better results.

Table 6 compares the DMOC low-thrust results with low-thrust reference trajectories from literature. These reference trajectories, denoted by LT ref 1 and LT ref 2, presented by Mingotti et al. [5], were created using a shooting optimization method. All trajectories begin in a $167 \mathrm{~km}$ circular orbit at the Earth and end in an elliptical orbit at the moon. Comparing the trajectories, DMOC trajectories require less fuel and flight time than the trajectories created using shooting methods. Because of similar end conditions, DMOC LT1-1 and DMOC LT1-2 may be compared directly with LT ref 1, and DMOC LT2-1 may be compared with LT ref 2. The mass fractions of DMOC LT1-1 and DMOC LT1-2 are an improvement of $46 \%$ and $42 \%$, respectively, over the mass fraction of LT ref 1 , and DMOC LT2-1 is a 59\% improvement over the mass fraction of LT ref 2. Also note the reduction in flight time for the DMOC solutions compared with the reference solutions. Furthermore, these comparisons exclude the small differences in $\Delta V_{E}$ necessary to start on the optimal trajectory. In addition, it should be noted that the optimal trajectories produced here are accurate to second order. For a higher fidelity solution, these solutions could act as initial guesses for a higher order method.

\section{Future Work}

The work presented here could be advanced through the use of more accurate models. For example, formulating an initial guess in the three-dimensional CR3BP and then optimizing it with DMOC based on the corresponding Lagrangian dependent on six variables would lead to more accurate and interesting results. Furthermore, the flexibility of DMOC allows for optimization using increasingly complicated models: elliptical orbits instead of circular, realistic inclinations, even ephemeris models. If an ephemeris model is used, the sun, Earth, and moon positions would be formulated as timevarying vectors. The low-thrust work could be improved by considering an optimal thrust direction, instead of simply applying tangential thrust. In that optimized case, it seems reasonable that an even smaller mass fraction would be achievable. Furthermore, rather than fixing $T_{t}$, the time at which low thrust is turned off, and the phase angle $\Phi$, these could be determined via an additional outer loop parameter optimization.

Because accuracy is of tantamount importance for trajectory design, further exploration of either higher order DMOC or timeadaptive DMOC is warranted. DMOC, as presented here, employs the midpoint rule to approximate the relevant integrals leading to second-order convergence. It is possible to use different quadrature rules to achieve higher levels of accuracy. It would be interesting to explore the effect of different quadrature rules on the solution accuracy and computation time. Furthermore, [45] describes timeadaptive DMOC, an indirect optimization method that adapts the step size according to the function $\sigma(q)$, dependent upon the state variables. The method is based on a discretization of Hamilton's principle applied to the Lagrangian of the optimal control problem. For certain problems, time-adaptive DMOC produces optimal solutions with smaller errors in both solution and energy evolution.

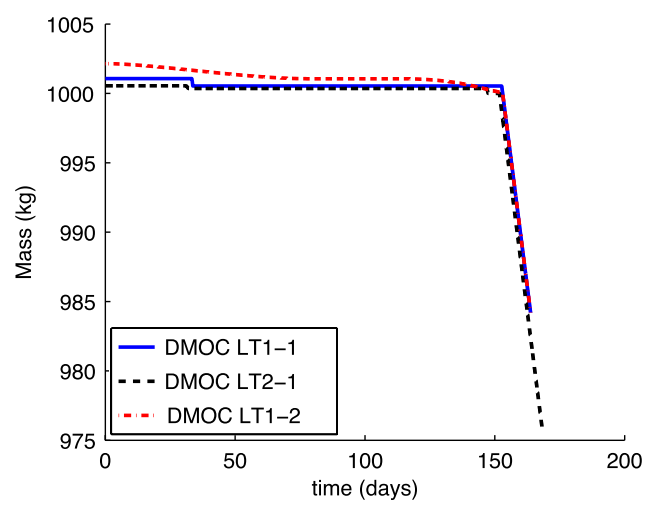

Fig. 19 Mass consumption for DMOC LT1-1, DMOC LT2-1, and DMOC LT1-2. 
At this time, only problems with $\sigma(q)$ have been tested, but the timedependent Earth-to-moon transfer described here would require $\sigma(q, t)$. Therefore, it is necessary to determine whether $\sigma(q, t)$ is valid for use with time-adaptive DMOC. Such an approach could produce more accurate solutions using less nodes and, consequently, less computation time.

\section{Conclusions}

The combination of dynamical systems theory with discrete mechanics and optimal control (DMOC) facilitates the design of interesting, low-energy trajectories from the Earth to the moon. Two different methods are effective for the creation of initial guess trajectories. The first method uses the invariant manifolds of the sunEarth and Earth-moon planar circular restricted three-body problems (PCR3BP), patching the two three-body systems together to generate a trajectory that begins at the Earth and is ballistically captured at the moon. Slight modification of the velocity at the intersection of the manifolds produces a trajectory valid for the four-body problem. The second method exploits the dynamics directly in the four-body problem, using the invariant manifolds of the PCR3BP as a guide. Next, the initial guess trajectories are optimized using DMOC, removing the impulsive midcourse change in velocity $\Delta V$ and reducing the $\Delta V$ necessary to leave Earth orbit and to circularize the orbit after the natural ballistic capture at the moon. Comparing the results achieved with different boundary constraints reveals that, when enforcing a final distance at the moon that is different than the initial guess, it is necessary to enforce the capture condition to maintain ballistic capture. Also, requiring the radial velocity to be zero at the endpoints is very important for reducing the $\Delta V$ to leave or enter circular orbits at the Earth and moon, respectively. The combination of invariant manifolds and DMOC successfully produces optimized trajectories with impulsive thrust from the Earth to the moon that are competitive with the literature, requiring up to $19 \% \Delta V$ less than a Hohmann transfer. DMOC is also shown to be very useful for the design of low-thrust trajectories. When compared with trajectories created with shooting methods, the DMOC trajectories demonstrate an improvement of up to $59 \%$ in the mass fraction and $22 \%$ in travel time. After an impulsive $\Delta V$ that sets the spacecraft on a trajectory influenced by the invariant manifolds of the sun-Earth and Earth-moon systems, less than $17 \mathrm{~kg}$ of fuel is required to reach an elliptical orbit at the moon.

\section{Acknowledgments}

This work was generously supported in part by the National Defense Science and Engineering Graduate Fellowship, the W. M. Keck Institute for Space Studies, and the Collaborative Research Centre 614 "Self-Optimizing Concepts and Structures in Mechanical Engineering" funded by the German Research Foundation (Deutsche Forschungsgemeinschaft) under grant SFB 614.

\section{References}

[1] Belbruno, E. A., and Miller, J. K., "Sun-Perturbed Earth-to-Moon Transfers with Ballistic Capture," Journal of Guidance, Control, and Dynamics, Vol. 16, No. 4, 1993, pp. 770-775. doi: $10.2514 / 3.21079$

[2] Belbruno, E. A., Capture Dynamics and Chaotic Motions in Celestial Mechanics, Princeton Univ. Press, Princeton, NJ, 2004.

[3] Camino, O., Alonso, M., Blake, R., Milligan, D., Bruin, J. D., and Ricken, S., "SMART-1: Europe's Lunar Mission Paving the Way for New Cost Effective Ground Operations (RCSGSO)," Sixth International Symposium "Reducing the Costs of Spacecraft Ground Systems and Operations (RCSGSO), European Space Agency ESA SP-601, Darmstadt, Germany, 2005.

[4] Koon, W. S., Lo, M. W., Marsden, J. E., and Ross, S. D., "Low Energy Transfer to the Moon," Celestial Mechanics and Dynamical Astronomy, Vol. 81, Nos. 1-2, 2001, pp. 63-73. doi:10.1023/A:1013359120468

[5] Mingotti, G., Topputo, F., and Bernelli-Zazzera, F., "Low-Energy, LowThrust Transfers to the Moon," Celestial Mechanics and Dynamical Astronomy, Vol. 105, Nos. 1-3, 2009, pp. 61-74. doi: $10.1007 / \mathrm{s} 10569-009-9220-7$
[6] Conley, C., "Low Energy Transit Orbits in the Restricted Three-Body Problem," SIAM Journal on Applied Mathematics, Vol. 16, No. 4, 1968, pp. 732-746. doi: $10.1137 / 0116060$

[7] McGehee, R., "Some Homoclinic Orbits for the Restricted Three-Body Problem," Ph.D. Dissertation, Univ. of Wisconsin, Madison, WI, 1969.

[8] Marsden, J. E., and Ross, S. D., "New Methods in Celestial Mechanics and Mission Design," American Mathematical Society Translations, Vol. 43, No. 1, 2005, pp. 43-73.

[9] Koon, W. S., Lo, M. W., Marsden, J. E., and Ross, S. D., Dynamical Systems, The Three-Body Problem, and Space Mission Design, Marsden Books, Wellington, New Zealand, 2008.

[10] Barden, B. T., Howell, K. C., and Lo, M. W., "Applications of Dynamical Systems Theory to Trajectory Design for a Libration Point Mission," Journal of the Astronautical Sciences, Vol. 45, No. 2, 1997, pp. 161-178.

[11] Gómez, G., Jorba, A., Masdemont, J., and Simó, C., "Study of the Transfer From the Earth to a Halo Orbit Around the Equilibrium Point $L_{1}$," Celestial Mechanics and Dynamical Astronomy, Vol. 56, No. 4, 1993, pp. 541-562. doi:10.1007/BF00696185

[12] Koon, W. S., Lo, M. W., Marsden, J. E., and Ross, S. D., "Heteroclinic Connections Between Periodic Orbits and Resonance Transitions in Celestial Mechanics," Chaos, Vol. 10, No. 2, 2000, pp. 427-469. doi:10.1063/1.166509

[13] Gómez, G., Koon, W., Lo, M., Marsden, J. E., Masdemont, J., and Ross, S. D., "Invariant Manifolds, The Spatial Three-Body Problem and Space Mission Design," Advances in the Astronautical Sciences, American Astronautical Society, Vol. 109, Pt. 1, pp. 3-22 AAS 013012001.

[14] Gawlik, E. S., Marsden, J. E., Campagnola, S., and Moore, A., "Invariant Manifolds, Discrete Mechanics, and Trajectory Design for a Mission to Titan," AAS/AIAA Space Flight Mechanics Meeting, American Astronautical Society Paper 09-226, 2009.

[15] Bosanac, N., Marsden, J. E., Moore, A., and Campagnola, S., "Titan Trajectory Design Using Invariant Manifolds and Resonant Gravity Assists," AAS/AIAA Space Flight Mechanics Meeting, American Astronautical Society Paper 10-170, 2010.

[16] Ross, S. D., and Scheeres, D. J., "Multiple Gravity Assists, Capture, and Escape in the Restricted Three-Body Problem," SIAM Journal on Applied Dynamical Systems, Vol. 6, No. 3, 2007, pp. 576-596. doi:10.1137/060663374

[17] Koon, W. S., Lo, M. W., Marsden, J. E., and Ross, S. D., "Resonance and Capture of Jupiter Comets," Celestial Mechanics and Dynamical Astronomy, Vol. 81, Nos. 1-2, 2001, pp. 27-38. doi:10.1023/A:1013398801813

[18] Williams, K. E., Barden, B. T., Howell, K. C., Lo, M. W., and Wilson, R. S., "GENESIS Halo Orbit Stationkeeping Design," International Symposium: Spaceflight Dynamics, Biarritz, France, June 2000.

[19] Koon, W. S., Lo, M. W., Marsden, J. E., and Ross, S. D., "The Genesis Trajectory and Heteroclinic Connections," Astrodynamics, Vol. 103, No. 3, 1999, pp. 2327-2343.

[20] Koon, W. S., Lo, M. W., Marsden, J. E., and Ross, S. D., "Shoot the Moon," Spaceflight Mechanics, Vol. 105, No. 2, 2000, pp. 1017-1030.

[21] Howell, K. C., and Kakoi, M., "Transfer Between the Earth-Moon and Sun-Earth Systems Using Manifolds and Transit Orbits," Acta Astronautica, Vol. 59, Nos. 1-5, 2006, pp. 367-380. doi:10.1016/j.actaastro.2006.02.010

[22] Mingotti, G., and Topputo, F., "Ways to the Moon: A Survey," AAS/ AIAA Space Flight Mechanics Meeting, American Astronautical Society Paper 11-283, 2011.

[23] Lo, M., The Interplanetary Superhighway and the Origins Program, IEEE Aerospace Conference Proceedings, IEEE Publications, Piscataway, NJ, Vol. 7, pp. 3543-3562, 2002.

[24] Lo, M. W., Anderson, R. L., Whiffen, G., and Romans, L., "The Role of Invariant Manifolds in Low Thrust Trajectory Design," AIAA/AAS Space Flight Mechanics Meeting, American Astronautical Society Paper 04-288, 2004.

[25] Davis, K. E., Anderson, R. L., Scheeres, D. J., and Born, G. H., "Connecting Libration Point Orbits of Different Energies Using Invariant Manifolds," AAS Space Flight Mechanics Meeting, American Astronautical Society Paper AAS 09-256, Savannah, Georgia, 2009.

[26] Marchand, B. G., Howell, K. C., and Betts, J. T., "Discrete Nonlinear Optimal Control of S/C Formations Near the L1 and L2 Points of the Sun-Earth/Moon System," AAS/AIAA Conference, American Astronautical Society Paper 05-341, 2005.

[27] Dellnitz, M., Ober-Blöbaum, S., Post, M., Schütze, O., and Thiere, B., "A Multi-Objective Approach to the Design of Low Thrust Space Trajectories Using Optimal Control," Celestial Mechanics and Dynamical Astronomy, Vol. 105, No. 1, pp. 33-59, 2009. 
[28] Dellnitz, M., Junge, O., Post, M., and Thiere, B., "On Target for Venus - Set Oriented Computation of Energy Efficient Low Thrust Trajectories," Celestial Mechanics and Dynamical Astronomy, Vol. 95, Nos. 1-4, 2006, pp. 357-370. doi:10.1007/s10569-006-9008-y

[29] Junge, O., Marsden, J. E., and Ober-Blöbaum, S., "Discrete Mechanics and Optimal Control," Proceedings of the 16th IFAC World Congress, Vol 16, No. 1, 2005. doi:10.1016/S0377-0427(00)00310-1

[30] Marsden, J. E., and West, M., "Discrete Mechanics and Variational Integrators," Acta Numerica, Vol. 10, 2001, pp. 357-514. doi:10.1017/S096249290100006X

[31] Ober-Blöbaum, S., "Discrete Mechanics and Optimal Control," Ph.D. Dissertation, University of Paderborn, Paderborn, Germany, 2008.

[32] Gill, P. E., Murray, W., and Saunders, M. A., "SNOPT: An SQP Algorithm for Large-Scale Constrained Optimization," Univ. of California, San Diego, CA, Rept. NA 97-2, 1997.

[33] Gill, P. E., Jay, L. O., Leonard, M. W., Petzold, L. R., and Sharma, V., "An SQP Method for the Optimal Control of Large-scale Dynamical Systems," Journal of Computational and Applied Mathematics, Vol. 120, Nos. 1-2, 2000, pp. 197-213. doi:10.1016/S0377-0427(00)00310-1

[34] Powell, M. J. D., "A Fast Algorithm for Nonlinearly Constrained Optimization Calculations," Numerical Analysis, edited by G. A. Watson, Vol. 630, Lecture Notes in Mathematics, Springer, New York, 1978, pp. 261-280.

[35] Han, S. P., "Superlinearly Convergent Variable-Metric Algorithms for General Nonlinear Programming Problems," Mathematical Programming, Vol. 11, No. 1, 1976, pp. 263-282. doi:10.1007/BF01580395

[36] Ross, S. D., "Cylindrical Manifolds and Tube Dynamics in the Restricted Three-Body Problem," Ph.D. Dissertation, California Inst. of Technology, Pasadena, CA, 2004.

[37] Betts, J. T., Practical Methods for Optimal Control Using Nonlinear Programming, Society for Industrial and Applied Mathematics,
Philadelphia, 2001.

[38] Marsden, J. E., and Ratiu, T., "Introduction to Mechanics and Symmetry", Texts in Applied Mathematics, Springer, New York, Vol. 17, 1994, pp. 205-210.

[39] Ober-Blöbaum, S., Junge, O., and Marsden, J. E., "Discrete Mechanics and Optimal Control: An Analysis," Control, Optimisation and Calculus of Variations, Vol. 17, No. 2, 2011, pp. 322-352. doi: $10.1051 / \mathrm{cocv} / 2010012$

[40] Betts, J. T., "Survey of Numerical Methods for Trajectory Optimization," Journal of Guidance, Control, and Dynamics, Vol. 21, No. 2, 1998, pp. 193-207.

doi: $10.2514 / 2.4231$

[41] Binder, T., Blank, L., Bock, H. G., Bulirsch, R., Dahmen, W., Diehl, M., Kronseder, T., Marquardt, W., Schlöder, J. P., and von Stryk, O., "Introduction to Model Based Optimization of Chemical Processes on Moving Horizons," Online Optimization of Large Scale Systems: State of the Art, edited by M. Grötschel, S. O. Krumke, and J. Rambau, Springer, Berlin, 2001, pp. 295-340.

[42] Moore, A., Ober-Blöbaum, S., and Marsden, J. E., "Optimization of Spacecraft Trajectories: A Method Combining Invariant Manifold Techniques and Discrete Mechanics and Optimal Control," AAS/AIAA Space Flight Mechanics Meeting, American Astronautical Society, Savannah, Georgia, 2009.

[43] Betts, J. T., and Huffman, W. P., "Mesh Refinement in Direct Transcription Methods for Optimal Control," Optimal Control Applications and Methods, Vol. 19, No. 1, 1998, pp. 1-21. doi:10.1002/(SICI) 1099-1514(199801/02)19:1<1::AID-OCA616> 3.0. $\overline{\mathrm{CO} ; 2-\mathrm{Q}}$

[44] Moore, A., Ober-Blöbaum, S., and Marsden, J. E., "Mesh Refinement Strategies for Spacecraft Trajectory Optimization Using Discrete Mechanics and Optimal Control," AAS/AIAA Space Flight Mechanics Meeting, American Astronautical Society Paper 11-127, 2011.

[45] Moore, A., "Discrete Mechanics and Optimal Control for Space Trajectory Design", Ph.D. Thesis, California Inst. of Technology, Pasadena, CA, 2011. 